\title{
Supersaturation in the Wyoming CCN Instrument
}

\author{
JEFFERSON R. SNIDER \\ Department of Atmospheric Science, University of Wyoming, Laramie, Wyoming \\ Markus D. PetTers \\ Department of Atmospheric Sciences, Colorado State University, Fort Collins, Colorado \\ Perry Wechsler and Peter S. K. Liu \\ Department of Atmospheric Sciences, University of Wyoming, Laramie, Wyoming
}

(Manuscript received 11 October 2005, in final form 23 February 2006)

\begin{abstract}
Two thermal diffusion cloud condensation nuclei (CCN) instruments were intercompared using a nearly monodisperse test aerosol composed of sodium chloride and ammonium sulfate. The main objective of this work is the determination of the maximum steady-state supersaturation within a thermal diffusion chamber. This required analysis of the uncertainties associated with the calibration of the instrument's plate temperature and light scattering measurement systems.

The fraction of test particles activating to form cloud droplets, the activated fraction, was evaluated over a range of dry particle diameters at four supersaturations. Values of both the particle size, corresponding to an activated fraction equal to 0.5 , and the width of a fitted activation function were derived. The former was used to evaluate the maximum steady-state chamber supersaturation. These studies reveal three significant findings. First, the derived chamber supersaturation is $\sim 40 \%$ smaller than values based on temperature measured at the top and bottom of the diffusion chamber. Second, using deliquesced test particles, mobility selected at a prescribed relative humidity, it is shown that the $40 \%$ discrepancy is not the result of a test particle shape effect. Third, the width of the activation function is substantially larger than the width of the test particle size spectra.

Plausible explanations for the $40 \%$ bias are considered. Contributors are the unintentional overestimation of the temperature difference imposed across the $\mathrm{CCN}$ chamber and the implicit assumption that water at the top and bottom of the $\mathrm{CCN}$ chamber has a vapor pressure equal to that over pure water.
\end{abstract}

\section{Introduction}

Deficiencies in scientific understanding of air pollution, and in particular its effect on cloud radiative properties, have spurred interest in the development of measurement techniques for a category of the atmospheric aerosol known as the cloud condensation nuclei (CCN). Measurement of the $\mathrm{CCN}$ provides a direct estimation of the propensity of the aerosol to form cloud droplets as a function of water vapor supersaturation (Rogers and Yau 1989). Commonly called the CCN

Corresponding author address: Jeff Snider, University of Wyoming, Dept. of Atmospheric Science, 1000 E. University Ave., Laramie, WY 82071-2000.

E-mail: jsnider@uwyo.edu activity spectrum, this property of the aerosol can also be derived from measurements of the aerosol size spectra, chemical composition, and mixing state (e.g., Covert et al. 1998). Comparison of indirect estimation of the activity spectrum with direct measurement constitutes a validation of the former, and this validation is most powerful if bias is accounted for in the direct measurement of the activity spectrum. Furthermore, comparisons conducted with an understanding of the measurement uncertainty, both random and systematic, can be used to establish reliable datasets for cloud model initialization (Brenguier 2003), and can also reveal the limitations of measurement systems ( $\mathrm{CCN}$ and aerosol) and thus motivate improvement of the techniques (Nenes et al. 2001; Roberts and Nenes 2005). These exercises are viewed as essential ingredients of research 

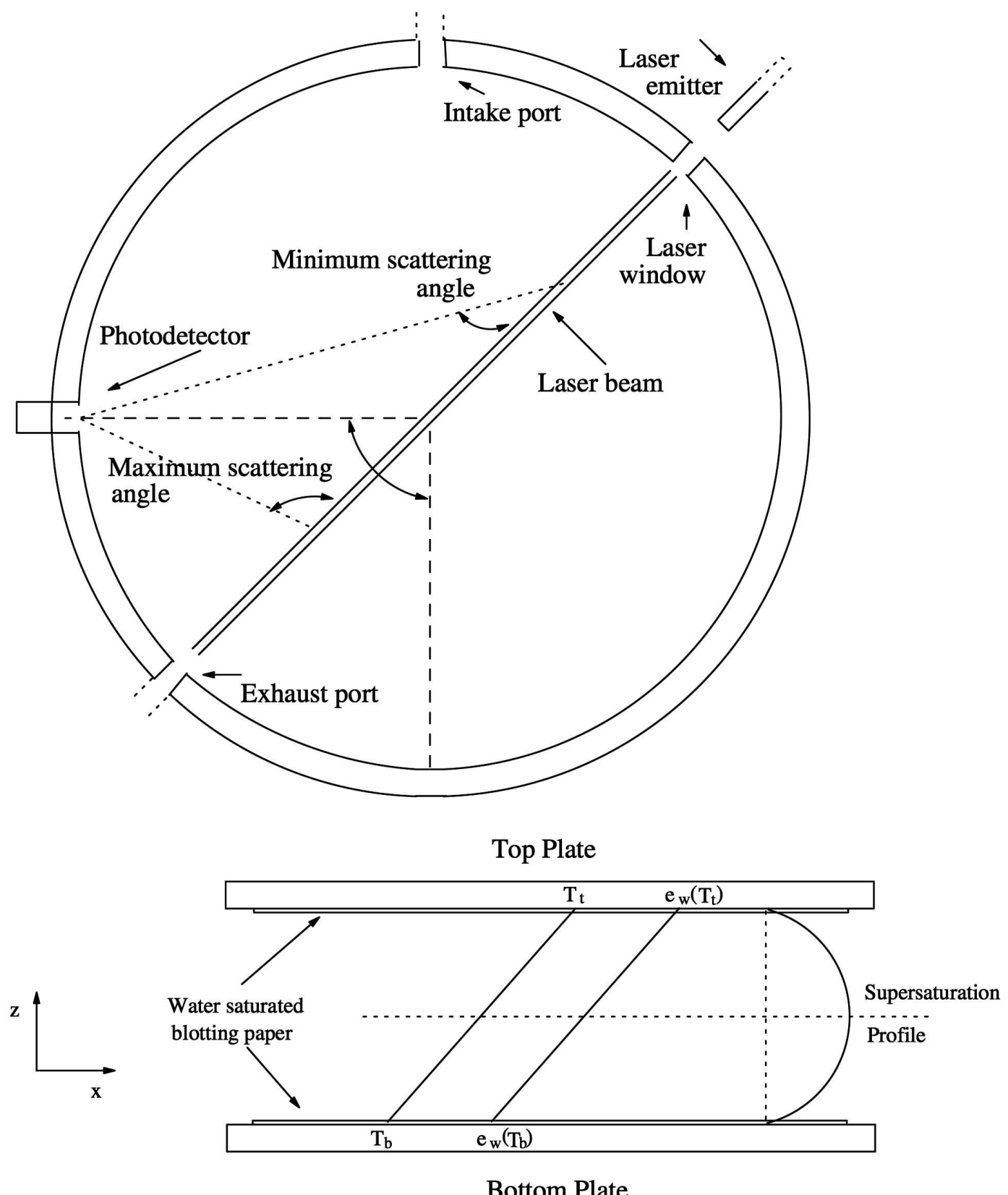

FIG. 1. Top and side views of the Wyoming thermal diffusion cloud chamber. The width to height ratio is 6:1; $e_{w}(T)$ is the $\mathrm{H}_{2} \mathrm{O}$ saturation vapor pressure.

aimed at improving understanding of the effects of aerosols on cloud radiative properties (Bates et al. 1998).

Here we report on laboratory studies aimed at improving understanding of the maximum water vapor supersaturation within a class of $\mathrm{CCN}$ instruments developed at the Department of Atmospheric Science at the University of Wyoming. This parameter is central to the issue of accurate $\mathrm{CCN}$ measurement, both for model validation and for basic research studies of heterogeneous water vapor condensation nucleation. Currently there are 12 of these instruments worldwide, operated both in research laboratories and on research aircraft.

The instrument (Fig. 1) is operated in a batch mode. The duty cycle starts with a 5-10-s interval during which the temperature of the bottom plate is stabilized by either heat addition or removal from the bottom of the 
chamber via a thermoelectric heat pump (the "wait interval"). This is followed by a 5-s interval when fresh aerosol is admitted to the chamber (the "flush interval"). Subsequently, the chamber is sealed for $20 \mathrm{~s}$ (the "detect interval"). During the detect interval a steadystate supersaturation profile, maximizing near the midplane of the chamber, is established. All particles with a critical supersaturation less than the maximum steady-state supersaturation $\left(S_{\max }\right)$ grow to supermicrometric size as activated cloud droplets. A data acquisition system is used to record laser light scattering from the centermost region of the chamber.

The laboratory studies discussed herein are used to establish the following three relationships: 1) between the signal voltage produced by two opposed thermocouples and the temperature difference imposed across the CCN chamber, 2) between the light scattering signal and activated droplet concentration, and 3) between the temperature difference and $S_{\max }$. We refer to these relationships as the plate temperature calibration, detector calibration, and the chamber calibration. Uncertainties associated with these calibrations are indicated by $\Delta, \sigma$, and $\delta$. Here $\Delta$ denotes a difference between two limits, and $\sigma$ is used to denote either a random error or the standard deviation of a distributed quantity. To distinguish the accuracy of a measurement we use the symbol $\delta$. Our nomenclature is defined further in the appendix.

Central to this study are our measurements of the fraction of nearly monodisperse test particles activating to form droplets (the activated fraction) and the fit of activated fraction versus test particle size (the activation function). We show that values of $S_{\max }$, derived from the size corresponding to $50 \% \mathrm{CCN}$ activation, are substantially smaller than those derived using a one-dimensional model of the thermal diffusion chamber. These studies also show that the width of the activation function is substantially larger than that anticipated for our test aerosols.

\section{Overview}

Schematic illustrations of the Wyoming thermal diffusion chamber are provided in Fig. 1. The top-plate temperature $\left(T_{t}\right)$ varies slowly with variations in the temperature of the instrument enclosure, and the bottom-plate temperature $\left(T_{b}\right)$ is controlled at a temperature colder than $T_{t}$ with a thermoelectric heat pump. The air gap between the two plates is $0.012 \mathrm{~m}$ and the plates are lined with wetted blotting paper, each with a thickness of $0.001 \mathrm{~m}$. Signal voltages produced by two opposed ${ }^{1}$ copper-constantan thermocouples embedded in the centers of the bottom and top plates (each with a thickness of $0.009 \mathrm{~m}$ ) are used to derive the difference between the top- and bottom-plate temperatures $T_{t}-$ $T_{b}$. Values of $T_{t}-T_{b}$, hereafter symbolized as $\Delta T$, and an independent measurement of the top-plate temperature are used to calculate $S_{\max }$ (Snider et al. 2003).

The calculation of $S_{\max }$ is based on the chamber model of Katz and Mirabel (1975). The following three assumptions are inherent to this approach: 1) light from the laser, positioned through the center of the chamber (Fig. 1), illuminates the region of maximum steadystate supersaturation; 2) $\mathrm{H}_{2} \mathrm{O}$ vapor pressure at the free surface of the blotter paper is the value expected for vapor in equilibrium with pure liquid water; and 3) the temperature difference measured by the plateembedded thermocouples accurately represents the temperature difference between the free surfaces.

Because the supersaturation is thought to maximize below the chamber midline, that is, $S_{\max }$ is shifted away from the midline toward the colder plate, the first assumption will bias estimations of $S_{\max }$. However, this bias is thought to be insignificant both because the calculated supersaturation profile is broad (Saxena et al. 1970), and because the laser beam (vertical height of $0.002 \mathrm{~m}$ ) illuminates both the middle of the chamber and the height corresponding to the predicted $S_{\max }$.

The second and third assumptions are not as easily justified. For the second we note that a $0.1 \%$ departure from the boundary condition assumed for the free surfaces, for example, resulting from saturation vapor pressure lowering by the blotting paper, has a profound effect on the value of $S_{\max }$ (Snider et al. 2003). Because of the sensitivity to the humidity boundary condition, and because conventional relative humidity sensors cannot distinguish between relative humidities between $100 \%$ and $99 \%$, much less between $100.0 \%$ and $99.9 \%$, humidity measurements cannot be used to validate the second assumption. The third assumption is necessary because accurate measurement of the free surface temperature difference is problematic.

The possibility of either a negative departure of the vapor pressure from the saturation value or of a temperature difference across the blotter paper lead us to

\footnotetext{
${ }^{1}$ The constantan leads of the thermocouples are welded and connected to circuit ground. The electromotive force between the copper leads is measured differentially, canceling the junction potentials generated at the circuit connections and minimizing radio frequency interference. The differential voltage is amplified to the signal voltage; both are proportional to the temperature difference between the two plates.
} 
conclude that a value of $S_{\max }$, derived using a measurement of $\Delta T$, is an upper-limit estimate of the actual maximum steady-state supersaturation. This suspicion is borne out by several studies (Leaitch et al. 1999; Snider et al. 2003; Bilde and Svenningsson 2004; Rissler et al. 2004). The work reported here examines the issue of $S_{\max }$ overestimation by the chamber model.

In Table 1 we present values of $\Delta T$ and the calculated values of $S_{\max }$. Also presented is a different set of $S_{\max }$ values, again based on $\Delta T$, but derived using a different formula for calculating $S_{\max }$. Both models are employed in presentations of CCN data (e.g., Fitzgerald 1972; Katz and Mirabel 1975; Brechtel and Kreidenweis 2000; Snider et al. 2003), with the contrast being presented here to remind readers that $S_{\max }$ is often based on a model of the chamber.

\section{a. Modifications of the Wyoming CCN instrument}

In the past, the Wyoming CCN instrument had a two-valve flow system. This arrangement led to interruption of the test aerosol stream during the wait and detect intervals, necessitating storage of test aerosol in a bag prior to sampling (Delene and Deshler 2000). To eliminate the bag sampling, we added a third solenoid valve (the bypass valve) that shunts the sample around the chamber during the wait and detect intervals. The sample volumetric flow rate through the instrument was fixed at either 3 or 2 actual liters per minute (ALPM) using a critical orifice. Addition of the bypass valve allowed us to sample directly from a steadyflowing test aerosol stream.

\section{b. Köhler model}

The Köhler model we utilize is described in Snider et al. (2003). The model is based on the Köhler equation developed by Chýlek and Wong (1998), water activitycomposition relationships from Tang and Munkelwitz (1994), solution density-composition relationships from Tang and Munkelwitz (1994), and a surface tension-temperature-composition relationship from Seinfeld and Pandis (1998). A particle temperature of $20^{\circ} \mathrm{C}$ was used for the calculations.

\section{Experimental}

The plate temperature calibration, detector calibration, and chamber calibration are discussed in this section. These form the basis for our analysis of the $\mathrm{CCN}$ concentration measurement uncertainties and for our comparisons of chamber model-based and Köhler model-based assessments of $S_{\max }$. For these investigations, we utilized two University of Wyoming CCN in-
TABLE 1. Plate temperature difference and $S_{\max }$.

\begin{tabular}{ccc}
\hline$\Delta T\left({ }^{\circ} \mathrm{C}\right)^{\mathrm{a}}$ & $S_{\max }(\%)^{\mathrm{b}}$ & $S_{\max }(\%)^{\mathrm{c}}$ \\
\hline 2.17 & 0.20 & 0.18 \\
3.05 & 0.40 & 0.35 \\
4.30 & 0.80 & 0.71 \\
6.03 & 1.60 & 1.41 \\
\hline
\end{tabular}

a Plate temperature difference $T_{t}-T_{b}(\Delta T)$ is presented with a numerical precision of $\pm 0.01^{\circ} \mathrm{C}$ to demonstrate the difference between the two estimates of $S_{\max }$.

${ }^{\mathrm{b}} S_{\max }$ based on the relationship between saturation vapor pressure $\left(e_{w}\right)$ and plate temperatures proposed by Katz and Mirabel (1975): $S_{\max }=100 \cdot\left(\left(\left(e_{w}\left(T_{t}\right)+e_{w}\left(T_{b}\right)\right) / 2\right) / e_{w}\left(\left(T_{t}+T_{b}\right) / 2\right)-1\right)$. The top-plate temperature is $20^{\circ} \mathrm{C}$.

${ }^{c} S_{\text {max }}$ based on the following relationship between saturation vapor density $\left(\rho_{w}\right)$ and plate temperatures: $S_{\max }=100 \cdot\left(\left(\left(\rho_{w}\left(T_{t}\right)+\right.\right.\right.$ $\left.\left.\left.\rho_{w}\left(T_{b}\right)\right) / 2\right) / \rho_{w}\left(\left(T_{t}+T_{b}\right) / 2\right)-1\right)$. The top-plate temperature is $20^{\circ} \mathrm{C}$.

struments, identified by serial numbers CCNC-100A104 and CCNC-100A-108 (hereafter CCN104 and CCN108, respectively). The measurements were conducted between April and October 2001, and between January 2002 and October 2003. As is discussed in detail in the following section, we refer to these time periods as the second Dynamics and Chemistry of Marine Stratocumulus (DYCOMS-II) and High Plains Cumulus $(\mathrm{HiCu})$ experiment eras.

Optical windows are located between the laser emitter and the chamber (Fig. 1) and between the detector and the chamber. These were cleaned once per day using cotton and isopropyl alcohol.

\section{a. Plate temperature calibration}

The plate temperature calibration was performed twice during the investigation period. In preparation for the DYCOMS-II experiment, conducted in July 2001 (Stevens et al. 2003), we based the plate temperature calibration on a signal voltage produced by the embedded top- and bottom-plate thermocouples and thermocouple reference functions (Burns et al. 1993). The calibration procedure was revised in 2002, in preparation for $\mathrm{HiCu}$, when we utilized both signal voltage from a second pair of thermocouples (the calibration thermometers; physically attached to the top and bottom surfaces of the plates), plus signal voltage from the embedded thermocouples, and reference functions to establish an updated calibration. Using heat flux predicted by the chamber model and the thermal conductivity of aluminum, we estimated the temperature difference across the half-thickness of a plate. This is $0.001^{\circ} \mathrm{C}$ for $S_{\max }=1.6 \%$ (Table 1) and smaller for smaller values of $S_{\max }$. This suggests that the DYCOMS-II and $\mathrm{HiCu}$ plate temperature calibrations 
should produce indistinguishable values of $\Delta T$ for the same signal voltages. In fact, these calibrations differed by $0.3^{\circ} \mathrm{C}$, and the direction of this difference was consistent with that inferred from examinations of the detector (section 4d) and the chamber calibrations (section $4 \mathrm{~h}$ ). This bias is thought to be due to uncompensated imperfections in the thermocouple connections made during the DYCOMS-II era. With regard to the $\Delta T$ measurement accuracy, we assign the value of $\delta_{T}=$ $0.2^{\circ} \mathrm{C}$ for the $\mathrm{HiCu}$ era data, which corresponds to the accuracy of temperature measurements made with the calibration thermometers.

\section{b. Detector calibration}

The detector calibration is a relationship between the concentration of activated cloud droplets and the light they scatter into the photodetector (Fig. 1). We establish this relationship by challenging a CCN instrument with test particles having a dry size larger than that necessary for activation. In that situation, concurrent measurements of the aerosol concentration can be used as a surrogate from the droplet concentration (Gras 1995; Delene and Deshler 2000). In this work the calibrating aerosol concentration measurement was made with a relative precision of $\pm 5 \%$ in a TSI 3010 condensation particle counter (CPC).

Test aerosols were generated by passing polydisperse populations, containing either sodium chloride or ammonium sulfate particles, through a TSI 3071 differential mobility analyzer (DMA). For reasons discussed in section $4 \mathrm{~b}$, the mobility-classified test aerosols are not absolutely monodisperse. A TSI scanning mobility particle sizer (SMPS) was used to monitor the size distribution generated by the 3071 DMA. The SMPS consists of a second DMA, with geometry the same as that of the 3071 DMA column, a controller platform (TSI 3080), and a TSI 3010 CPC. The duration of the SMPS scan was $285 \mathrm{~s}$ and size spectra (particle concentration per unit decimal logarithmic size interval versus mean size) were generated from mobility spectra using the TSI inversion routine.

We use standard nomenclature to describe the airflows in the two DMAs: the polydisperse, sheath, excess, and monodisperse flows. The monodisperse-tosheath flow ratios were controlled at 1:5 and 1:10 in the 3071 and SMPS DMAs, respectively. Relative humidities ( $\mathrm{RH}$; measurement is discussed in section 3d) associated with the 3071 and SMPS excess streams were measured and always smaller than 10\%.

The aerosol stream exiting the 3071 DMA was mixed with a dried 6-ALPM dilution airstream $(\mathrm{RH}<10 \%)$ and passed through a charge neutralizer $\left({ }^{210} \mathrm{Po}\right.$, Aerosol Dynamics, Inc.). The airstream exiting the neutral- izer was split into flows entering the calibrating $\mathrm{CPC}$, the two CCN instruments, and the SMPS. Particle size was set at 0.14 and $0.16 \mu \mathrm{m}$ for the sodium chloride and ammonium sulfate detector calibrations, respectively. These sizes are sufficiently large to promote activation at the center of the chamber even if it is operated at the smallest supersaturation utilized in this study $(0.2 \%$; see Snider et al. 2003).

Following Delene and Deshler (2000), we refer to the difference between the maximum CCN photodetector signal during the 20-s detect interval and the minimum signal during the 5-s flush interval as the peak detector voltage $\left(V_{\text {peak }}\right)$. The calibrating $\mathrm{CCN}$ concentration was measured at the end of the 5-s flush interval.

\section{c. Chamber calibration via the dry-selection technique}

In the following two sections we describe techniques used to create the nearly monodisperse test aerosols utilized in the chamber calibration, that is, the calibration that determines the Köhler model-based estimations of $S_{\max }$. We refer to these as the "dry selection" and the "wet selection" techniques. These techniques allow us to probe the question of whether particle asphericity, or the presence of air or water occlusions within the test particles, introduces an appreciable bias in the determination of $S_{\max }$. Test particle concentrations were comparable for both techniques with typical concentration values equal to $500 \mathrm{~cm}^{-3}$.

In what we refer to as the dry-selection technique, aqueous sodium chloride and ammonium sulfate solutions were atomized (TSI 3075 constant output atomizer), dried to RH $=40 \%$ (TSI 3062 diffusion dryer), brought to charge equilibrium (TSI 3012 aerosol neutralizer), and mobility classified (TSI 3071 DMA). The RH of the sheath airstream was less than $10 \%$. While operating the $\mathrm{CCN}$ instruments at one of four plate temperature differences (Table 1), the dry particle size was set at values ranging from 0.02 to $0.16 \mu \mathrm{m}$. CCNand CPC-based concentrations $(\mathrm{CCN}$ and $\mathrm{CN}$, respectively) were evaluated, with the former being derived from $V_{\text {peak }}$ via the detector calibration. As is described in greater detail in section $4 \mathrm{f}$, this approach yields an assessment of the dry size corresponding to $\mathrm{CCN} / \mathrm{CN}=$ 0.5 , which we symbolize as $D_{50}$. Values of $D_{50}$ derived from calibrations performed with ammonium sulfate test particles were used to evaluate $S_{\max }$ via application of the Köhler model. In the case of dry-selected sodium chloride test particles, a shape correction was applied to the $D_{50}$ (section $4 \mathrm{a}$ ), and the shape-corrected value of $D_{50}$ was used to derive $S_{\text {max }}$, again via application of the Köhler model.

With the exception of the valve change (section 2a), 
the addition of the Aerosol Dynamics Inc. (ADI) neutralizer (section $3 b$ ), and the sodium chloride shape correction (section $4 \mathrm{a}$ ), the approach outlined here is the same as that utilized by Snider et al. (2003).

\section{d. Chamber calibration via the wet-selection technique}

The setup used for what we refer to as the wetselection technique is illustrated in Fig. 2. Here the RH of the polydisperse stream is set at $89 \%$, via passage through humidifier 1 , and the $\mathrm{RH}$ of the sheath airstream is controlled at $65 \%$ by a Sable Systems DG-1 dewpoint generator (humidifier 2). The RH of the polydisperse, sheath, excess, and the monodisperse airstreams were monitored using Vaisala HMP230 RH sensors. These measurement locations are indicated as RH-1, RH-2, RH-3, and RH-4. The RH measurements are made with an accuracy of $\pm 2 \%$ (Vaisala 2001). The $\mathrm{RH}$ of the polydisperse airstream (89\%, location RH-1) was sufficient for particle deliquescence prior to classification in the 3071 DMA. These particles are expected to behave as spheres, and the size extracted from the 3071 DMA $\left(D_{\text {wet }}\right)$ plus the RH measured in the excess airstream were used to derive the sphere-equivalent dry diameter $D_{\text {se }}$ via our Köhler model. This is illustrated in Fig. 3.

A complication associated with the wet-selection technique is the time dependence of $\mathrm{RH}$, experienced by particles transiting the 3071 DMA. Sensitivity of our results to this phenomenon was explored by estimating the position of the interface between the polydisperse/ sheath air mixture and the unmixed sheath air. This interface position was formulated as

$$
X=\sqrt{2 D_{v} t}
$$

where $D_{v}=3.3 \times 10^{-5} \mathrm{~m}^{2} \mathrm{~s}^{-1}$ is the water vapor diffusivity at the temperature and pressure of our experiments $\left(21^{\circ} \mathrm{C}\right.$ and $\left.780 \mathrm{hPa}\right)$, and $t$ is time.

The RH values predicted by Eq. (1) are shown in Fig. 4. Here it is evident that $0.2 \mathrm{~s}$ is required for the $\mathrm{RH}$ associated with the polydisperse-sheath mixture to decrease from $89 \%$ to $69 \%$. Because this time interval is two orders of magnitude larger than required for $D_{\text {wet }}$ to adjust to a $20 \% \mathrm{RH}$ step change (Lewis and Schwartz 2004), we assume that particles in the 3071 DMA adjust instantaneously to local values of the RH. The particle trajectories in Fig. 4 were derived by numerically integrating the equation of motion (Knutson and Whitby 1975) subject the instantaneous RH adjustment assumption and the Köhler model. For the upper trajectory (dashed) we assumed a constant a relative humidity trajectory. For this simulation, the $\mathrm{RH}$ is set at

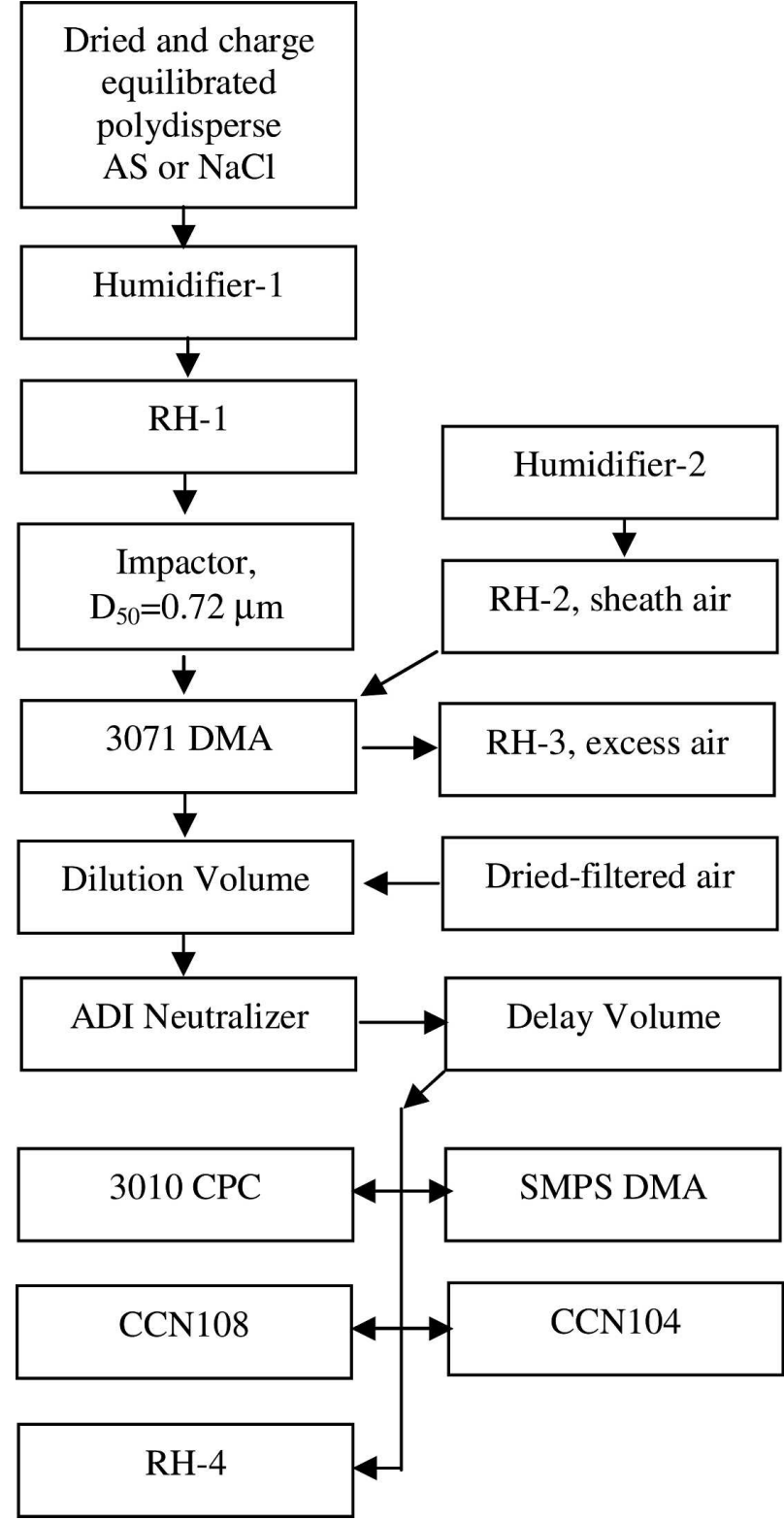

FIG. 2. Experimental setup used for chamber supersaturation studies conducted via the wet-selection technique.

the value that results from homogenization of the polydisperse and sheath airstreams (69\%, i.e., the RH value observed at sensor location RH-3). The lower trajectory (solid) corresponds to the time-dependent RH field illustrated by the coloration in Fig. 4. Our calculations indicate that the assumption of constant RH leads to a $2 \%$ bias in the value of $D_{\text {se }}$ derived from $D_{\text {wet }}$ and RH-3. Because Eq. (1) is only an approximation of the RH field within the 3071 DMA, and because the $2 \%$ bias is small relative to particle sizing uncertainties ( $\pm 4 \%$, section $4 \mathrm{a}$ ), we do not account for this bias in our analysis. 


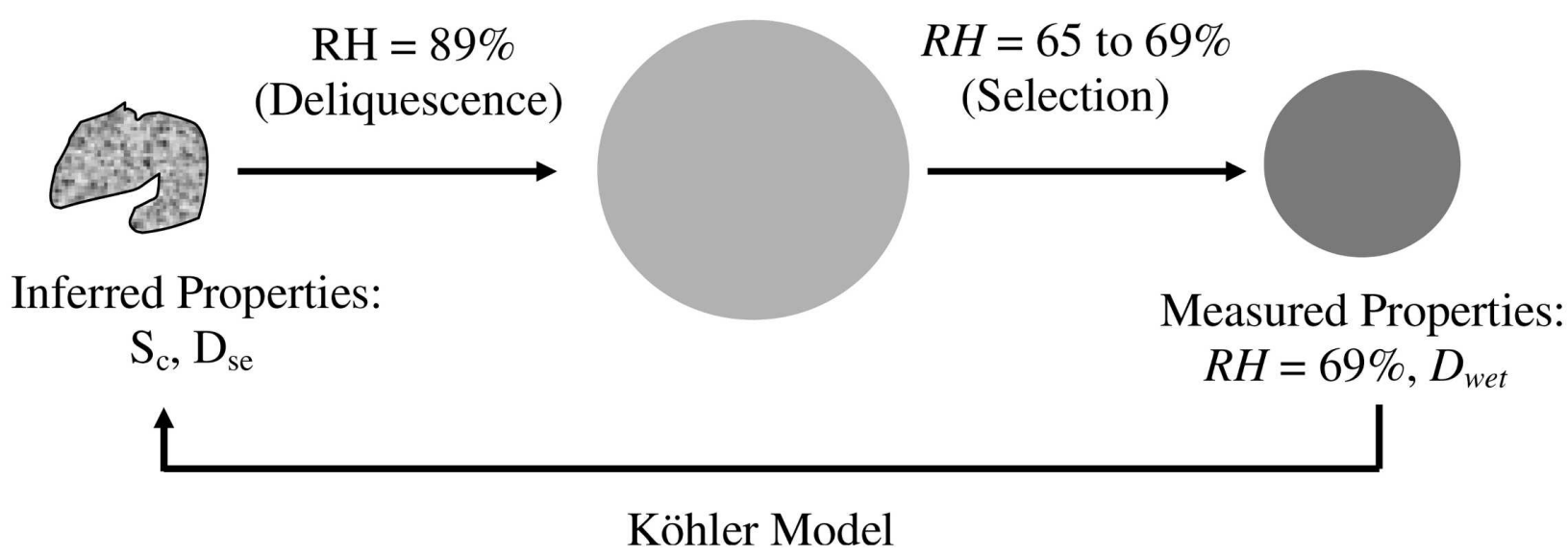

FIG. 3. Illustration of the measured and derived properties in the wet-selection experiments. Here $S_{c}$ is the critical supersaturation corresponding to the sphere-equivalent dry size $\left(D_{\mathrm{se}}\right)$. The latter is derived, via the Köhler model, from the measurement of RH in the excess flow in the 3071 DMA and the wet size $\left(D_{\text {wet }}\right)$.

In summary, the wet-selection technique circumvents the ambiguity inherent to the dry-selection technique because it does not rely on the assumption that the mobility-equivalent dry size, for selection in the 3071 DMA, is equal to the sphere-equivalent dry size. The wet-selection technique, however, is not without its own limitations, stemming from the time history of $\mathrm{RH}$ in the 3071 DMA, and as such cannot be used to make a completely unbiased determination of $D_{50}$. In spite of this, we shall see that the two techniques yield experimentally indistinguishable values of $S_{\max }$.

\section{Results}

Here we define the nomenclature and assumptions used in the presentation of the data, and then present the results. For the dry-selection experiments employing ammonium sulfate test particles, results are presented in terms of a mobility-equivalent dry diameter $\left(D_{\mathrm{me}}\right)$ on the assumption that this is equal to the sphere-equivalent dry diameter $\left(D_{\mathrm{se}}\right)$. In contrast, for the dry-selection experiments, performed using sodium chloride, we shape correct the $D_{\text {me }}$ to $D_{\text {se }}$ using a derived $D_{\mathrm{se}} / D_{\text {me }}$ ratio. Our calculation assumes a dynamic shape factor equal to 1.08 for our dry-selected sodium chloride particles, assumed to be cubical (Hinds 1999), and employs the formulation presented by Gysel et al. [2002, their Eq. (7)] to arrive at $D_{\mathrm{se}} / D_{\mathrm{me}}=0.96$ for all dry particle diameters of relevance to our study (0.02$0.16 \mu \mathrm{m})$. For the wet-selection experiments, and for both salts, we present the chamber calibration data in terms of $D_{\text {se }}$, derived from measurements of $D_{\text {wet }}$ and RH (Fig. 3). Finally, in our discussion of the chamber calibration we refer to two different chamber super- saturations: 1) the "nominal supersaturation" $\left(S_{\text {nom }}\right)$, derived with a value of $\Delta T$ via the Katz and Mirabel chamber model, and 2) the effective supersaturation $\left(S_{\text {eff }}\right)$, derived from $D_{50}$ via the Köhler model.

\section{a. Sizing comparison-Wet and dry selection}

Because test particle size accuracy is an important aspect of this work, we begin with an examination of the particle sizing data. Table 2 provides a statistical summary of ratios of two sizes: 1) the diameter selected in the 3071 DMA, and 2) the mode diameter corresponding to a SMPS size spectrum. These ratios were derived for 85 size spectra collected during both the detector and chamber calibration studies. Mode diameters ranged from 0.02 to $0.16 \mu \mathrm{m}$. The distinction between dry and wet selection, both in Table 2 and in general, is one of either employing the two humidifiers shown in Fig. 2 (wet selection), or not (dry selection). In either case the SMPS DMA sampled dilution-dried aerosol $(\mathrm{RH}<10 \%)$, so in the case of the dry-selection experiments (i.e., the second and third columns of Table 2) the summary constitutes a direct comparison of sizing by the two DMAs. For the dry-selection experiments, using either sodium chloride or ammonium sulfate, the SMPS and 3071 DMA sizes agree within $\pm 2 \%$, on average, and on a case-by-case basis the compared sizes never differ by more than $4 \%$. A conservative estimate of the relative sizing uncertainty is $\pm 4 \%$ and this is used in subsequent discussions and in our uncertainty analysis.

Results of the wet-selection experiments, for ammonium sulfate test aerosol (fourth column of Table 2), indicate that the relationship between the SMPSmeasured mobility-equivalent dry size and the sphere- 


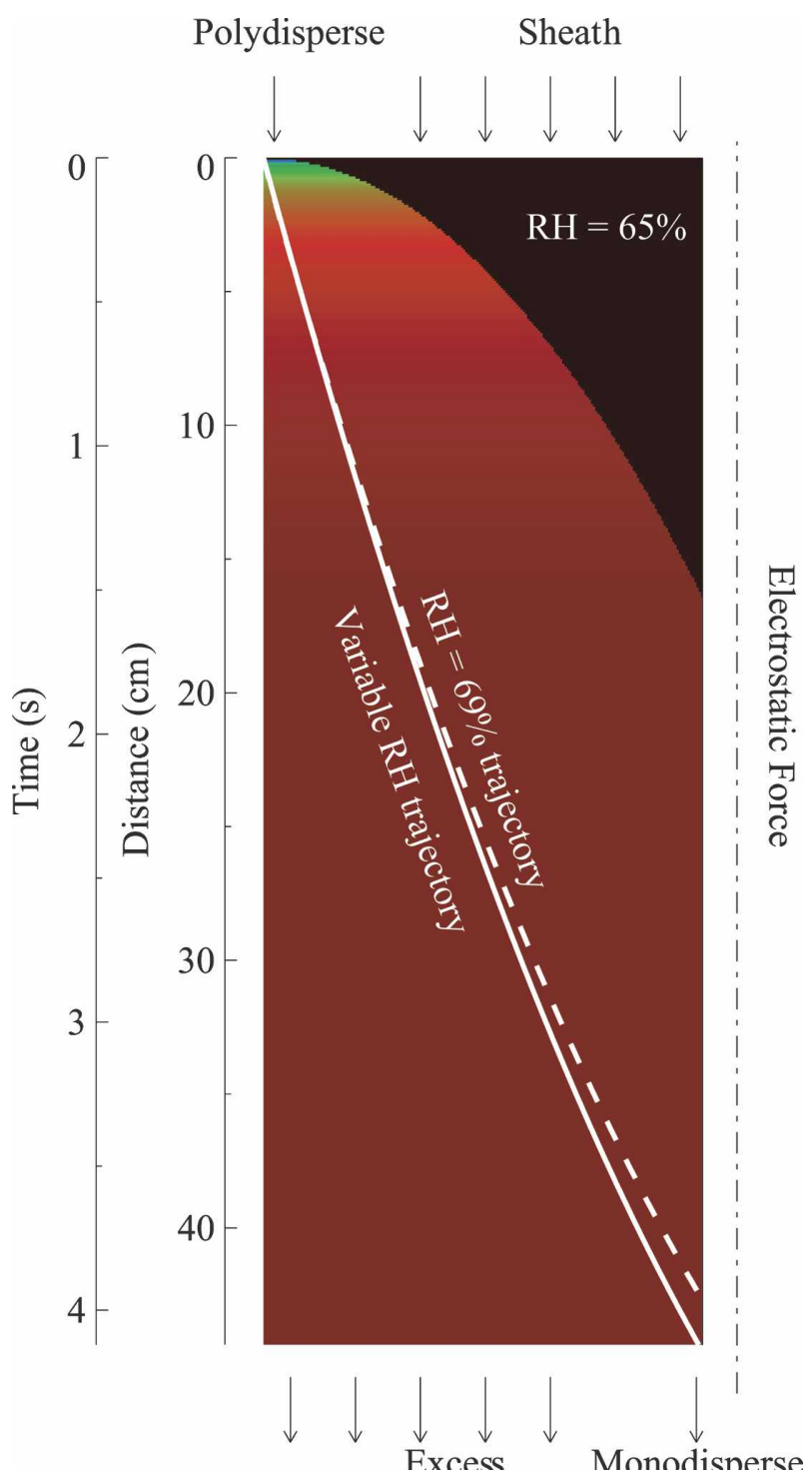

Relative Humidity (\%)

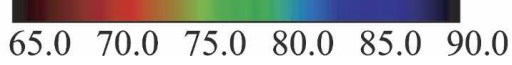

FIG. 4. Humidity distribution in the 3071 DMA during the wetselection experiments. The dashed line indicates a particle trajectory assuming constant RH (69\%). The solid line indicates a particle trajectory assuming the same initial dry size as for the dashed trajectory but accounting for a path-dependent RH.

equivalent dry size (defined by the wet-selection technique; Fig. 3) is not complicated by shape effects or the presence of voids or water in these particles. That is, our ammonium sulfate test aerosols behaved in a manner consistent with the assumption that these particles, subsequent to dilution drying (Fig. 2), are effluoresced and are compact spheres. To the contrary, the average value of the size ratio shown in the fifth column of Table 2 is consistent with the conjecture that the wet- selected sodium chloride particles, subsequent to dilution drying, did not behave as compact sodium chloride spheres. We attribute this behavior solely to a shape effect and account for it with a $D_{\mathrm{se}} / D_{\text {me }}$ ratio equal to 0.96 (section 4a). This correction is larger than that presented in the fifth column of Table 2, but the difference (0.96 versus 0.94$)$ is small in comparison to the $\pm 4 \%$ sizing error.

\section{b. Size spectra-Wet and dry selection}

Dry size spectra measured by the SMPS DMA, corresponding to both the wet- and dry-selection technique, are shown for ammonium sulfate in Figs. 5a and $5 \mathrm{~b}$, and for sodium chloride in Figs. $6 \mathrm{a}$ and $6 \mathrm{~b}$. The top panels are for $0.060-\mu \mathrm{m}$ particles dry selected in the 3071 DMA and the bottom panels are for wet particles, characterized by a $0.060-\mu \mathrm{m}$ sphere-equivalent dry diameter, selected in the 3071 DMA via the wet-selection technique (Fig. 3). In both instances, the particle stream exiting the 3071 DMA was dilution dried to $\mathrm{RH}<10 \%$ and brought to charge equilibrium, prior to sampling in the SMPS DMA. These procedures are described in detail in sections $3 \mathrm{c}$ and $3 \mathrm{~d}$. The modes corresponding to +1 and +2 charge-state particles (i.e., when these particles are at the exit of the 3071 DMA, prior to reestablishment of charge equilibrium) appear at approximately 0.06 and $0.09 \mu \mathrm{m}$, respectively. Henceforth we refer to the +1 and +2 modes as the "singlet mode" and "doublet mode," respectively.

The following two plotting techniques are utilized in Figs. 5a, 5b, 6a, and 6b: 1) the size axis is logarithmic, and 2) the singlet mode amplitude is used to normalize the spectra. This allows us to interpret the area below the doublet mode portion of the plotted curve, centered at $0.09 \mu \mathrm{m}$, as the relative contribution of the doublet mode particles to the total concentration. The spectra demonstrate that the doublet mode particles make a small contribution to the total. For our SMPS dataset the maximum, average, and standard deviation of the doublet-to-singlet mode concentration ratio are 0.12 , 0.08 , and 0.02 , respectively $(n=85)$.

Figures $5 \mathrm{a}, 5 \mathrm{~b}$, and $6 \mathrm{a}$ show the size selected by the 3071 DMA as a vertical blue tick, and the $\pm 4 \%$ relative sizing uncertainty as a horizontal blue rectangle. Comparisons of the selected and singlet mode sizes demonstrate that there is agreement within the sizing uncertainty. In Fig. $6 \mathrm{~b}$ the 3071 DMA size indicated by the vertical blue tick was derived by taking the ratio of the sphere-equivalent dry diameter $(0.060 \mu \mathrm{m})$, corresponding to wet selection in the 3071 DMA, and the $D_{\text {se }} / D_{\text {me }}$ ratio (section 4a). Again, there is reasonable agreement between the anticipated and measured mode sizes. 
TABLE 2. Statistical summary of size ratios.

\begin{tabular}{|c|c|c|c|c|}
\hline & \multicolumn{2}{|c|}{ Dry-selection 3071/SMPS size ratio* } & \multicolumn{2}{|c|}{ Wet-selection 3071/SMPS size ratio** } \\
\hline & Ammonium sulfate & Sodium chloride & Ammonium sulfate & Sodium chloride \\
\hline Average & 0.99 & 0.99 & 0.99 & 0.94 \\
\hline Standard deviation & 0.02 & 0.02 & 0.02 & 0.01 \\
\hline Maximum & 1.04 & 1.03 & 1.01 & 0.98 \\
\hline Minimum & 0.97 & 0.97 & 0.97 & 0.91 \\
\hline Number & 26 & 31 & 6 & 22 \\
\hline
\end{tabular}

* Diameter ratio corresponding to dry-selection experiments. See text for details.

** Diameter ratio corresponding to wet-selection experiments. See text for details.

For our investigations of the activation function (section 4f) we require a generalized formulation for the width of the 3071 DMA size spectra. The latter is here defined as product of a constant (to be determined) multiplied by the base width of the singlet mode. The base width is

$$
\Delta_{\mathrm{DMA}}=\Psi\left(Z_{+1}+\Delta Z / 2\right)-\Psi\left(Z_{+1}-\Delta Z / 2\right),
$$

where $\Psi$ represents the transformation from electrical mobility to diameter (sphere assumed), $\Delta Z$ is the base width of the DMA transfer function, and $Z_{+1}$ is the electrical mobility corresponding to the selected size. According to DMA theory (Knutson and Whitby 1975) the relative half-width $\Delta Z /\left(2 Z_{+1}\right)$ is equivalent to the monodisperse-to-sheath flow ratio (1:5 in our experiments; section 3b), and based on this and the selected size, and Eq. (2), values of the base width were calculated. These are compared to the width of the SMPS size spectra in Figs. 5a, 5b, 6a, and 6b. Here the calculated base width $\left(\Delta_{\mathrm{DMA}}\right)$ is represented by the horizontal red rectangle. This calculation was repeated for the 85 SMPS spectra and compared to widths of the singlet mode at a relative amplitude of 0.61 . By doing this, we are approximating the singlet mode size distribution as Gaussian, although in fact it is known to be triangular. The resulting relationship between the Gaussian width and the calculated base width is

$$
2 \sigma_{\mathrm{DMA}}=\gamma \Delta_{\mathrm{DMA}} .
$$

Here the factor of 2 comes from the fact that the Gaussian width, at amplitude equal to 0.61 , is equivalent to 2 times its standard deviation. For our data the constant $\gamma$ evaluated to $0.52 \pm 0.03(n=85)$, which is close to the value expected for the triangular DMA transfer function (0.39). The fact that $\gamma$ exceeds the value anticipated is consistent with sources of broadening resulting from particle diffusion and other nonidealities. Based on Eq. (64) in Flagan (1999), and a monodisperse-tosheath flow ratio (1:5), we anticipate that diffusional broadening is most pronounced at diameters smaller than $0.04 \mu \mathrm{m}$. We did find that $\gamma$ increases with decreasing size for experiments conducted at mode sizes smaller than $0.04 \mu \mathrm{m}$, but not to a degree larger than the scatter of $\gamma$ over the $0.02-0.16-\mu \mathrm{m}$ size range. We therefore use a size-independent value for $\gamma$ throughout this study.

\section{c. Detector calibration-Measurement}

Figure 7 shows the relationship between $V_{\text {peak }}$ and CPC-measured particle concentrations at two nominal supersaturations ( $S_{\text {nom }}=0.2$ and $0.8 \%$ ). It illustrates an ambiguity arising from the photodetection technique, that is, the same $\mathrm{CN}$ concentration yields a range of $V_{\text {peak }}$ values. The $V_{\text {peak }}$ variability results from the small sample volume of the Wyoming instrument, from the random location of droplets within that volume, and from the fact that droplets scatter light in a more forward direction, that is, those droplets more distant from the detector divert more light into the detector compared to those scattering from closer to the detector. The photodetector sample volume is defined by the segment of the laser bounded by the maximum and minimum scattering angles (Fig. 1). The Poisson error lines shown in the bottom two panels of Fig. 7 were formulated as $\mathrm{CN}=\alpha V_{\text {peak }} \pm \sqrt{\vartheta \alpha V_{\text {peak }}}$, where the photodetector sample volume $\left(\vartheta=0.16 \mathrm{~cm}^{3}\right)$ is based on the work of Delene and Deshler (2000). In addition to utilizing CPC-based concentration data for their detector calibration, Delene and Deshler (2000) also derived concentrations by counting droplets within a prescribed sample volume (the video sample volume). For such application, the utility of Poisson counting statistics is obvious, but this is not necessarily applicable to the photodetection technique. In lieu of the Poisson error, we now propose a new method for estimating the detector calibration uncertainty.

Using the CPC-based detector calibration data, we now define "percentile calibration lines" of the form

$$
\mathrm{CN}=\alpha_{i} V_{\text {peak }} .
$$



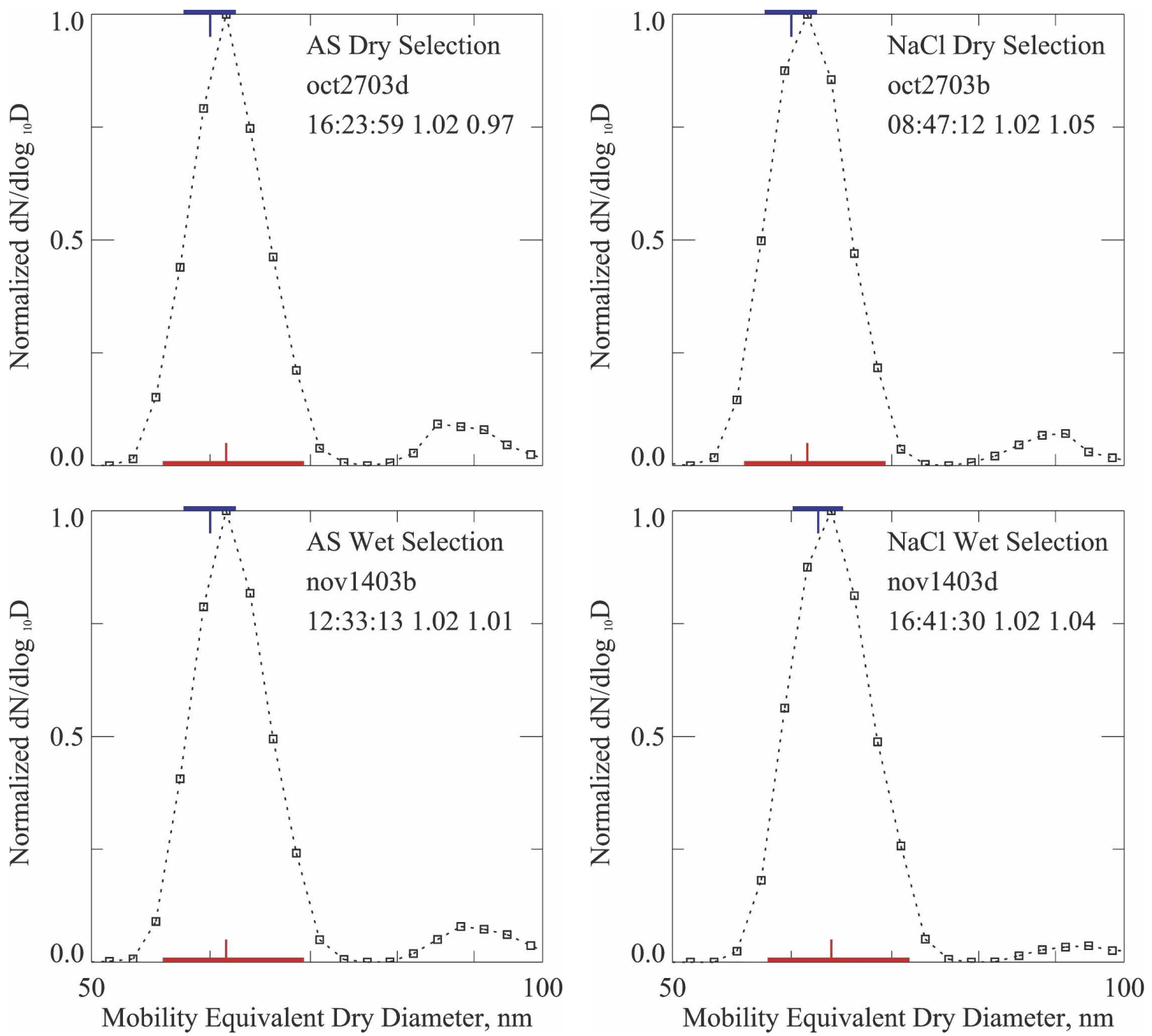

FIG. 5. Normalized SMPS DMA size spectra for ammonium sulfate test aerosols, measured at $\mathrm{RH}<10 \%$, vs the decimal logarithm of the mobility-equivalent dry diameter. The singlet and doublet mode particles appear at approximately 60 and 90 $\mathrm{nm}$, respectively. The vertical blue ticks indicate the singlet mode dry size anticipated for (top) dry selection made in the 3071 DMA and (bottom) wet selection made in the 3071 DMA. The width of the horizontal blue rectangle corresponds to the $\pm 4 \%$ particle sizing uncertainty. The vertical red ticks indicate the mode size corresponding to singlet particles and the width of the horizontal red rectangle indicates the width anticipated by DMA theory. See text for details.

Here $\mathrm{CN}$ is the condensation nucleus concentration measured by the calibrating CPC, $V_{\text {peak }}$ is the peak photodetector signal, and $\alpha_{i}$ is a detector calibration line defined such that $i \%$ of the data are below the fitted line. The colored lines shown in the top two pan-

FIG. 6. As in Fig. 5, but for (top) dry-selected and (bottom) wet-selected sodium chloride. In the bottom panel, the size indicated by the vertical blue tick was derived by taking the ratio of the sphere equivalent dry diameter $\left(D_{\text {se }}\right)$, selected in the 3071 DMA, and the $D_{\mathrm{se}} / D_{\mathrm{me}}$ ratio (0.96). See text for details.

els of Fig. 7 represent the 50th percentile result for individual detector calibrations performed during the DYCOMS-II era. Data from these individual calibrations were pooled and fitted, and the resulting 50th percentile line was used to define the DYCOMS-II era detector calibration. The fit is indicated by the dashed $\alpha_{50}$ lines shown in bottom panels. Plotted above this is the 84th percentile line, below is the 16th percentile line, and nearly coinciding to these are the Poisson error lines derived using the detector sample volume reported by Delene and Deshler (2000). Clearly, the per- 

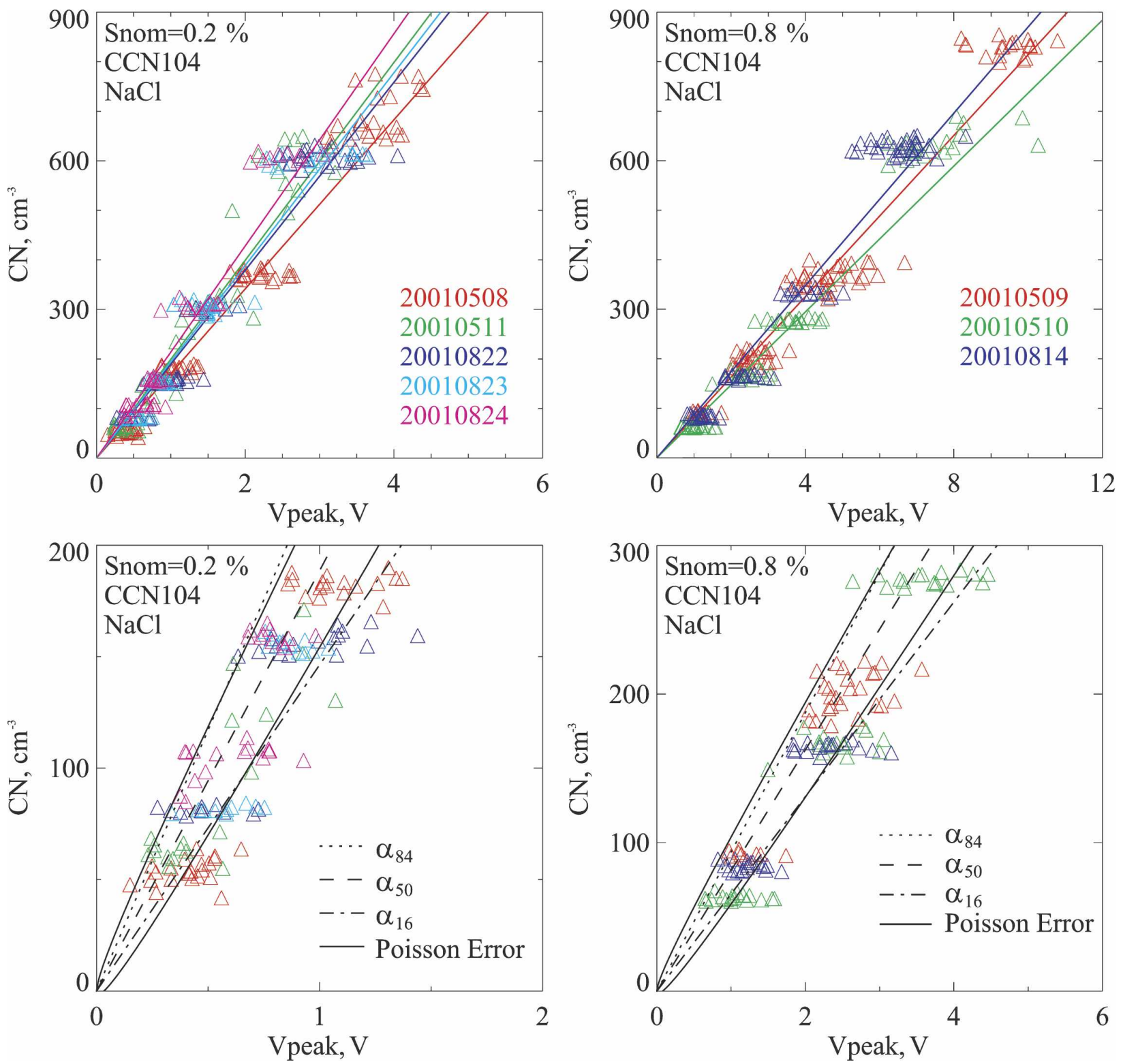

FIG. 7. Detector calibration results for (left) CCN104 at $S_{\text {nom }}=0.2 \%$ and (right) $S_{\text {nom }}=0.8 \%$. Both correspond to DYCOMS-II era data. (top) The spread of $\alpha_{50}$ for individual calibrations are shown; dates of the laboratory experiments are indicated in year/month/day format. (bottom) A blowup of the left-hand corner of the top panels and the $\alpha_{50}$ line corresponding to a fit of all of the individual detector calibration data; also, the 84th and 16th percentile error lines $\left(\alpha_{84}\right.$ and $\left.\alpha_{16}\right)$ and the Poisson error for a detector sample volume $\vartheta=0.16 \mathrm{~cm}^{3}$ are shown.

centile and Poisson error limits are in reasonable agreement.

\section{d. Detector calibration-Fitting}

Figure 8 shows the $\alpha_{50}$ values (triangles) plotted versus nominal supersaturation $\left(S_{\text {nom }}\right)$. Decreasing $\alpha_{50}$ with increasing supersaturation results because $V_{\text {peak }}$ depends on droplet size, and thus droplet growth rate, and because the latter varies in proportion to supersaturation. This inverse $\alpha_{50} / S_{\text {nom }}$ relationship is dis- cussed by Oliveira and Vali (1995), Delene and Deshler (2000), and Snider et al. (2003). In contrast to the fitting model utilized by those investigators, we find a power law of the form $\alpha_{50}=a S_{\text {nom }}^{b}$ to be an improved descriptor of the $\alpha_{50} / S_{\text {nom }}$ relationship. Our fits are indicated by the solid lines shown in Fig. 8. The red dotted lines are fits of the DCYOMS-II era $\alpha_{84}$ and $\alpha_{16}$ values. They are described by power laws of the form $\alpha_{84}=$ $(a+\Delta a) S_{\text {nom }}^{b-\Delta b}$ and $\alpha_{16}=(a-\Delta a) S_{\text {nom }}^{b+\Delta b}$, with $\Delta a$ defined as the largest of the two differences between the 

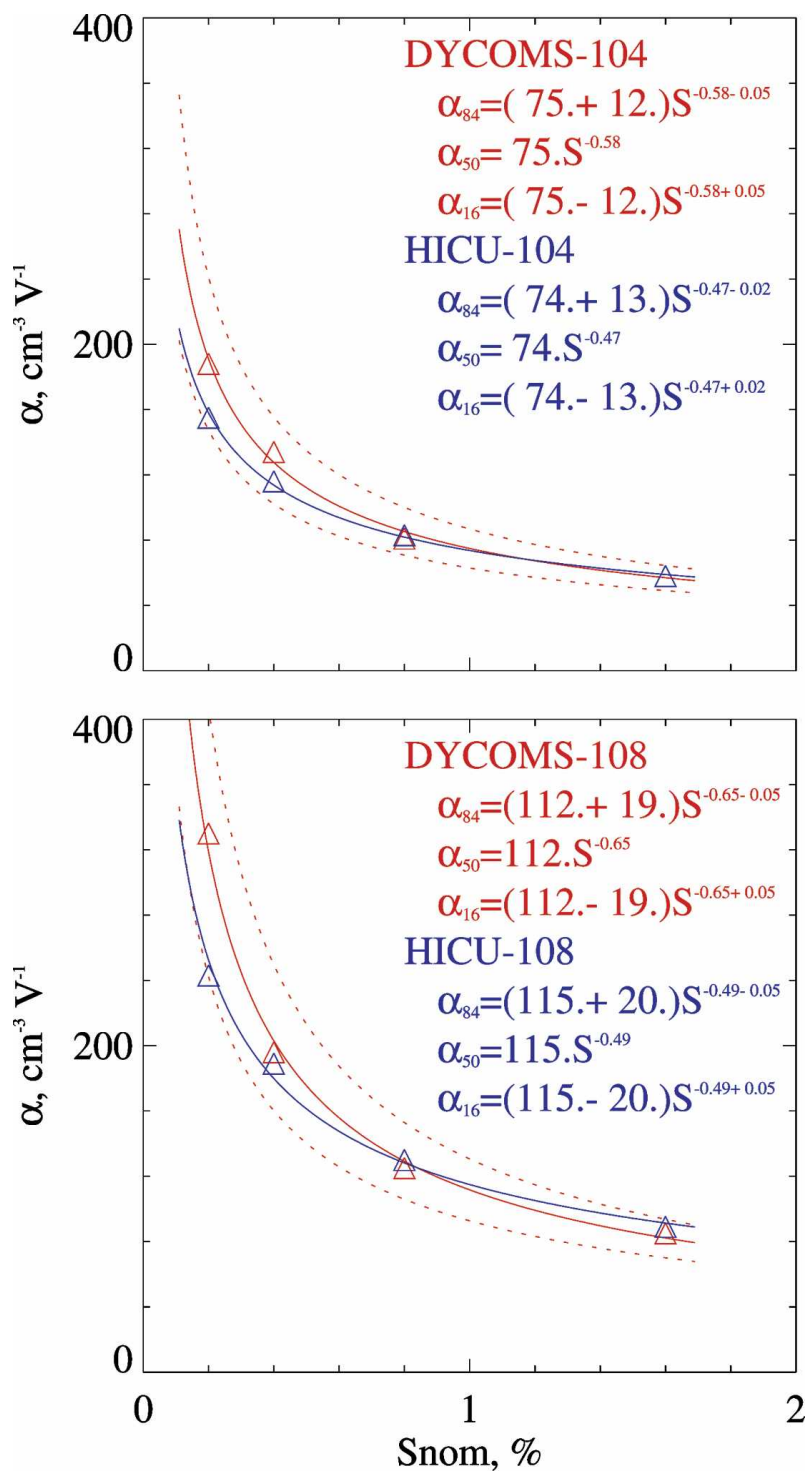

FIG. 8. Detector calibration results for (top) CCN104 and (bottom) CCN108. Triangles correspond to the DYCOMS-II and $\mathrm{HiCu}$ era $\alpha_{50} / S_{\text {nom }}$ relationships. Solid red and blue lines are the $\alpha_{50}=a S_{\text {nom }}^{b}$ fits. Dashed red lines indicate fits of the DYCOMS-II era $\alpha_{84}$ (upper dashed red line) and $\alpha_{16}$ (lower dashed red line) values. The equations illustrate the coefficients $a, b, \Delta a$, and $\Delta b$. See text for details.

" $a$ " values derived by fitting either $\alpha_{84} / S_{\text {nom }}$ and $\alpha_{50} /$ $S_{\text {nom }}$, or $\alpha_{50} / S_{\text {nom }}$ and $\alpha_{16} / S_{\text {nom. }}$. The parameter $\Delta b$ is defined in a similar manner.

Figure 8 and Table 3 demonstrate a shift in the detector calibration between DYCOMS-II and $\mathrm{HiCu}$ eras. This is largest for $S_{\text {nom }}=0.2 \%$, where the $\mathrm{HiCu}$ value plots at the DYCOMS-II value minus one standard deviation. We think this shift is traceable to the plate temperature calibration, and in section $4 \mathrm{~h}$, we present evidence in support of this suspicion. On the
TABLE 3. Detector calibration results: $S_{\text {nom }}$ is the nominal chamber supersaturation; $\alpha_{50}$ is the detector calibration coefficient; $\sigma_{\alpha}$ is the detector calibration uncertainty defined by Eq. (5); $\delta_{\alpha}$ is the standard deviation of $\alpha_{50}$, derived from individual chamber calibrations; $\mathrm{NC}=$ standard deviation not calculated because the number of individual $\alpha_{50}$ values is less than four; $n$ is the number of individual $\alpha_{50}$ values.

\begin{tabular}{lcccc}
\hline \hline \multicolumn{5}{c}{ CCN104-DYCOMS-II } \\
\hline$S_{\text {nom }}(\%)$ & 0.2 & 0.4 & 0.8 & 1.6 \\
$\alpha_{50}\left(\mathrm{~cm}^{-3} \mathrm{~V}^{-1}\right)$ & 191 & 128 & 85 & 57 \\
$\sigma_{\alpha}\left(\mathrm{cm}^{-3} \mathrm{~V}^{-1}\right)$ & 50 & 28 & 15 & 7 \\
$\delta_{\alpha}\left(\mathrm{cm}^{-3} \mathrm{~V}^{-1}\right)$ & 16 & $\mathrm{NC}$ & $\mathrm{NC}$ & $\mathrm{NC}$ \\
$n$ & 5 & 2 & 3 & 2 \\
\hline \multicolumn{5}{c}{$\mathrm{CCN} 108-\mathrm{DYCOMS}-\mathrm{II}$} \\
\hline$S_{\mathrm{nom}}(\%)$ & 0.2 & 0.4 & 0.8 & 1.6 \\
$\alpha_{50}\left(\mathrm{~cm}^{-3} \mathrm{~V}^{-1}\right)$ & 319 & 203 & 129 & 82 \\
$\sigma_{\alpha}\left(\mathrm{cm}^{-3} \mathrm{~V}^{-1}\right)$ & 89 & 47 & 24 & 11 \\
$\delta_{\alpha}\left(\mathrm{cm}^{-3} \mathrm{~V}^{-1}\right)$ & 69 & $\mathrm{NC}$ & $\mathrm{NC}$ & $\mathrm{NC}$ \\
$n$ & 4 & 2 & 2 & 3 \\
\hline & $\mathrm{CCN} 104-\mathrm{HiCu}$ & & \\
\hline$S_{\mathrm{nom}}(\%)$ & 0.2 & 0.4 & 0.8 & 1.6 \\
$\alpha_{50}\left(\mathrm{~cm}^{-3} \mathrm{~V}^{-1}\right)$ & 158 & 114 & 82 & 59 \\
$\sigma_{\alpha}\left(\mathrm{cm}^{-3} \mathrm{~V}^{-1}\right)$ & 35 & 23 & 15 & 10 \\
$\delta_{\alpha}\left(\mathrm{cm}^{-3} \mathrm{~V}^{-1}\right)$ & 20 & 10 & 10 & 5 \\
$n$ & 7 & 7 & 6 & 6 \\
\hline & $\mathrm{CCN} 108-\mathrm{HiCu}$ & & \\
\hline$S_{\mathrm{nom}}(\%)$ & 0.2 & 0.4 & 0.8 & 1.6 \\
$\alpha_{50}\left(\mathrm{~cm}^{-3} \mathrm{~V}^{-1}\right)$ & 252 & 180 & 128 & 91 \\
$\sigma_{\alpha}\left(\mathrm{cm}^{-3} \mathrm{~V}^{-1}\right)$ & 66 & 40 & 23 & 13 \\
$\delta_{\alpha}\left(\mathrm{cm}^{-3} \mathrm{~V}^{-1}\right)$ & 67 & 16 & 10 & 7 \\
$n$ & 7 & 7 & 6 & 6 \\
\hline
\end{tabular}

assumption that the detector calibration shift is attributable to a shift in the plate temperature calibration, we estimated the latter via a two-step process. First, the DYCOMS-II value of $\alpha_{50}$, evaluated at $S_{\text {nom }}=0.4 \%$ (Fig. 8), was shifted horizontally to intersect the $\mathrm{HiCu}$ data-fitting curve. Second, the $S_{\text {nom }}$ values at the intersection were input into the Katz and Mirabel chamber model (section 2). These calculations indicated that the DYCOMS-II value of $\Delta T$ is $0.5^{\circ} \mathrm{C}$ smaller than that based on the DYCOMS-II plate temperature calibration.

\section{e. Detector calibration-Uncertainty}

We define the detector calibration uncertainty $\sigma_{\alpha}$ as the vertical distance between the $\alpha_{84}$ and the $\alpha_{50}$ lines (Fig. 8). Formally, the detector calibration uncertainty is expressed as

$$
\sigma_{\alpha}\left(S_{\mathrm{nom}}\right)=(a+\Delta a) S_{\mathrm{nom}}^{b-\Delta b}-a S_{\mathrm{nom}}^{b},
$$

where $a, b \Delta a$, and $\Delta b$ are the fitted coefficients described in section $4 \mathrm{~d}$. Values of $\sigma_{\alpha}$ are presented in 
Table 3. At $S_{\text {nom }}=0.2 \%$, the detector calibration uncertainty can be as large as $25 \%$ of the detector calibration coefficient $\left(\alpha_{50}\right)$. Two processes are thought to contribute to this uncertainty. The first is stochastic and originates from the random placement of droplets within the scattering volume and from the angular asymmetry of the scattering. The second is systematic and is evident in the standard deviations of the individual $\alpha_{50}$ values. These standard deviations, symbolized by $\delta_{\alpha}$, are presented in Table 3 . The observation that $\delta_{\alpha}$ is comparable to $\sigma_{\alpha}$ implies that a portion of the uncertainty originates from systematic change from one detector calibration instance to another. A plausible explanation is that this change results from lightattenuating materials either adhered to the surfaces of the optical system, or removed from those surfaces by our daily cleaning with isopropyl alcohol (section 3). We have not investigated this hypothesis but we do take the value $2 \delta_{\alpha}$ to be the magnitude of a detector calibration bias and use this to estimate error that propagates into the chamber calibration from the detector calibration (section $4 \mathrm{~h}$ ).

\section{f. Chamber calibration-Measurement}

As we discussed previously, the objective of the chamber calibrations is the evaluation of the particle size corresponding to $50 \%$ activation (i.e., $\mathrm{CCN} / \mathrm{CN}=$ $0.5)$. Results from dry- and wet-selection experiments conducted at $S_{\text {nom }}=0.2 \%$, using sodium chloride aerosols, are shown in Fig. 9. Recall that the mobilityselected sodium chloride dry diameters were converted to sphere-equivalent dry diameters by multiplying by a $D_{\text {se }} / D_{\text {me }}$ ratio (section 4 a).

The triangles shown in Fig. 9 represent mean CCN/ $\mathrm{CN}$ ratios (activated fractions), each corresponding to between 10 and 20 pairs of $\mathrm{CCN}$ and $\mathrm{CN}$ measurements, and the vertical bars indicate plus and minus one standard deviation of that mean. Actual fitting of the activated fraction data was conducted using the individual CCN measurements, each normalized by a companion $\mathrm{CN}$ measurement. Sets of these activated fraction measurements, each consisting of at least 45 measurements of the $\mathrm{CCN} / \mathrm{CN}$ ratio, were fitted to a cumulative Gaussian

$$
A(D)=\frac{1}{\sigma_{50}} \frac{1}{\sqrt{2 \pi}} \int_{0}^{D} \exp \left[-\left(D^{\prime}-D_{50}\right)^{2} /\left(2 \sigma_{50}^{2}\right)\right] d D^{\prime} .
$$

Here $A(D)$ is the activation function, $\sigma_{50}$ is the standard deviation of that function, and $D_{50}$ is the diameter defining $50 \%$ activation. The parameter $\sigma_{50}$ should be
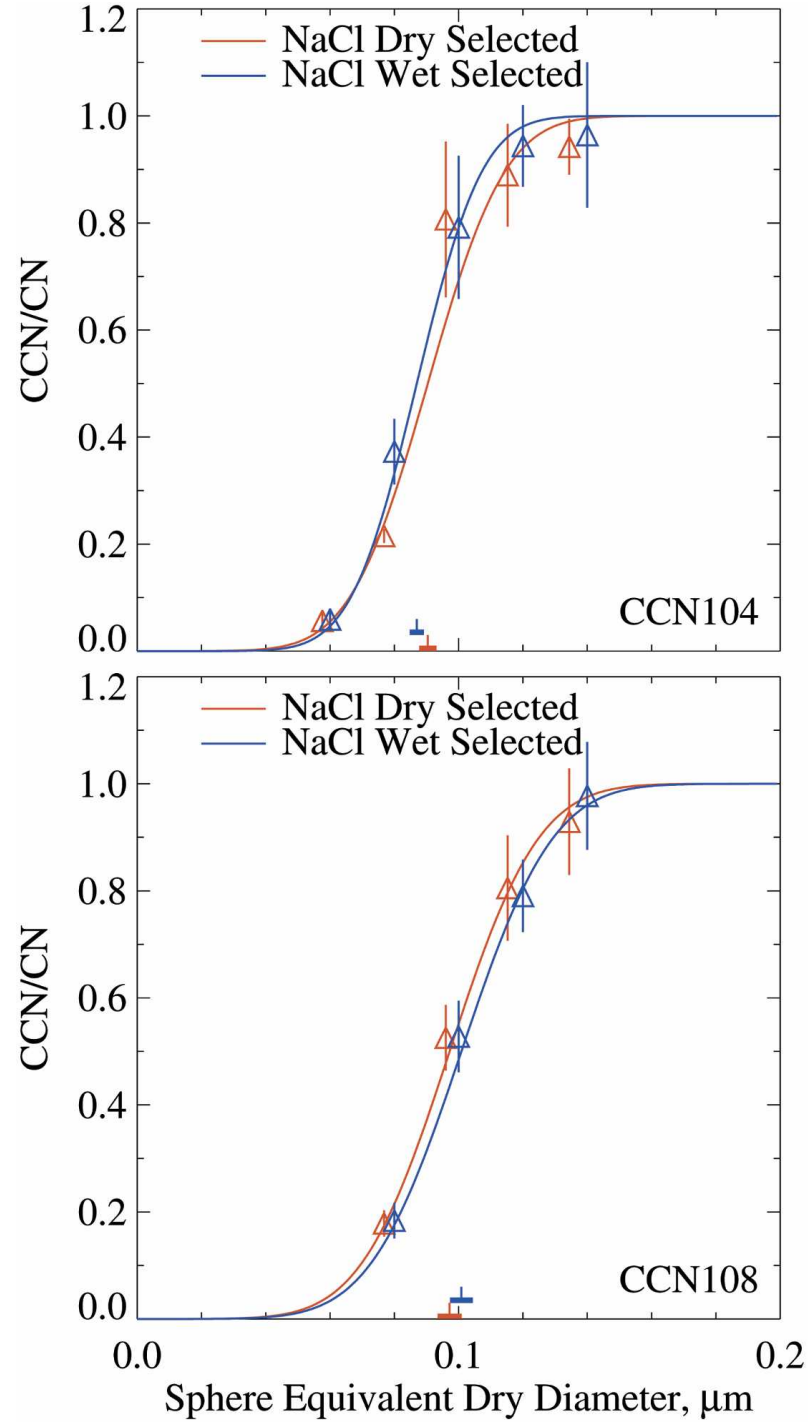

FIG. 9. Results of two wet- and two dry-selection chamber calibration experiments conducted at $S_{\text {nom }}=0.2 \%$ : (top) CCN104 and (bottom) CCN108. Triangles represent $\mathrm{CCN} / \mathrm{CN}$ means; vertical lines show plus and minus one standard deviation of the means. Values of $D_{50}$ are indicated by the vertical ticks (red or blue) plotted near the abscissa. Estimated $D_{50}$ accuracies are indicated by the width of the horizontal rectangle, also shown near the abscissa beneath the vertical ticks. The horizontal rectangle extends from $D_{50}-2 \delta_{50}$ to $D_{50}+2 \delta_{50}$. See text for details.

distinguished from the fitting uncertainty associated with $D_{50}$, which we discuss next.

The sets of activated fraction and particle diameter were fitted to Eq. (6) using a procedure known as "curvefit" (Interactive Data Language; RSI, Inc.). Statistical weights passed to the procedure were derived using the random error component of the detector calibration (section $4 \mathrm{e}$ ) and the $\mathrm{CN}$ (section $3 \mathrm{~b}$ ) random error. The procedure returns a standard deviation associated with 
the fitted value of $D_{50}$; we symbolize this as $\delta_{50}$. Figure 9 presents $D_{50}$ plus or minus 2 times $\delta_{50}$ as horizontal colored rectangles, near the abscissa, and values of $D_{50}$ as vertical colored ticks extending upward from the horizontal rectangles. The actual uncertainty associated with $D_{50}$ is expected to be larger than $2 \delta_{50}$. First, we have not accounted for random error associated with particle size, but in relative terms this is small compared to the random error component of the detector calibration (sections $4 \mathrm{a}$ and $4 \mathrm{e}$, respectively). Second, we have assumed that Eq. (6) is a good model for the activated fraction data. This is suspect because on average $8 \%$ of the particles selected by the 3071 DMA are apportioned into a doublet mode characterized by particle size larger than the singlet mode particles (section $4 b$ ). Therefore, $2 \delta_{50}$ is thought to represent a lower-limit estimate of the accuracy of $D_{50}$.

\section{g. Chamber calibration-Broadening of the distribution}

Figure 10 presents the 32 pairs of $D_{50}$ and $2 \sigma_{50}$ values, 16 for each $\mathrm{CCN}$ instrument, with results coming from both the DYCOMS-II and $\mathrm{HiCu}$ eras. It is apparent that $\sigma_{50}$, the standard deviation of the activation function, increases with increasing $D_{50}$. This is consistent, at least qualitatively, with DMA theory (Knutson and Whitby 1975) and the fact that the monodisperse-tosheath flow ratio was constant for our studies (section $3 b$ ). Here we focus on a comparison of $2 \sigma_{50}$ (i.e., the width of the activation function) and the width of the size spectra produced by the 3071 DMA. Recall that the latter can be expressed as a constant $(\gamma=0.52)$ times the base width anticipated by DMA theory [Eqs. (2) and (3)].

The black line shown in Fig. 10 represents the size dependence of $\gamma \Delta_{\mathrm{DMA}}$, that is, the width for our 3071 DMA at a relative amplitude equal to 0.61. Assuming that this width defines the minimum possible width of the activation function, the comparison of $\gamma \Delta_{\mathrm{DMA}}$ and $2 \sigma_{50}$ is compelling evidence for activation fraction broadening occurring within the $\mathrm{CCN}$ instrument. This conclusion does not preclude the possibility that a portion of the broadening comes from the presence of doublet mode particles, but we do not think that these particles can explain all of the broadening. Further studies are needed for investigating this issue.

\section{h. Chamber calibration-Discussion}

Chamber calibration results are presented in Fig. 11 as a ratio of the Köhler model-based and chamber model-based supersaturations, that is, $S_{\text {eff }}$ and $S_{\text {nom }}$, respectively. The ratios are stratified by $\mathrm{CCN}$ instru-

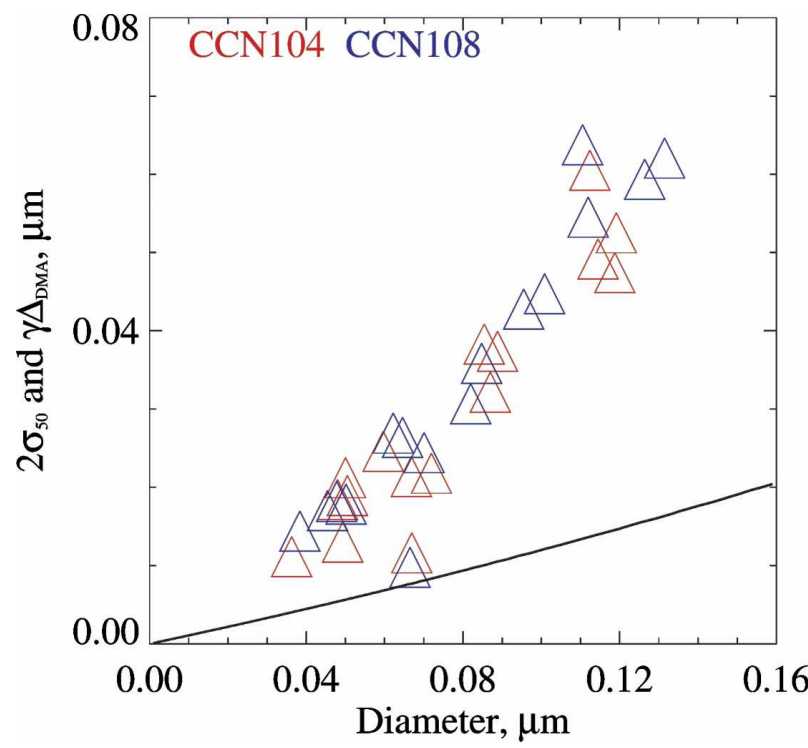

FIG. 10. Comparison of the width of the activation function $\left(2 \sigma_{50}\right.$; triangles $)$ and the width of the size spectrum $\left(\gamma \Delta_{\mathrm{DMA}}\right.$; black line), both vs particle diameter. Size dependence of $\Delta_{\mathrm{DMA}}$ is based on Knutson and Whitby (1975), the monodisperse-to-sheath flow ratio (1:5), a mean free path for air $(0.088 \mu \mathrm{m})$ in Laramie, WY $(P=780 \mathrm{hPa})$, and a Cunningham slip correction factor [Eq. (8.34) in Seinfeld and Pandis 1998].

ment, era (DYCOMS-II results are circled), salt, particle selection technique, and $S_{\text {nom }}$. We also present upper-limit $S_{\text {eff }} / S_{\text {nom }}$ estimates. These were evaluated by perturbing the $S_{\text {eff }} / S_{\text {nom }}$ ratio upward in response to two suspected biases. The first involves a reassessment of $S_{\text {eff }}$ at a smaller size $\left(D_{50}-2 \delta_{50}\right.$; section $\left.4 f\right)$ and the second involves calculation of $S_{\text {nom }}$ at smaller values of $\Delta T$. Our $\Delta T$ perturbation is either $0.5^{\circ} \mathrm{C}$ for the DYCOMS-II era data (section $4 \mathrm{~d}$ ) or the $\Delta T$ measurement accuracy for the $\mathrm{HiCu}$ era data $\left(\delta_{T}=0.2^{\circ} \mathrm{C}\right.$, section 3a). Combined, these biases do not push any of the $S_{\text {eff }} / S_{\text {nom }}$ ratios beyond unity.

With the exception of the two measurements plotting at $S_{\text {eff }} / S_{\text {nom }}=0.8$, the comparison of results derived using the dry- and wet-selection technique indicate agreement within $0.2 S_{\text {eff }} / S_{\text {nom }}$ units. Again excluding the $S_{\text {eff }} / S_{\text {nom }}=0.8$ values, there is no indication of a systematic $S_{\text {eff }} / S_{\text {nom }}$ shift in a comparison of the two techniques. This indicates that neither particle shape effects (i.e., asphericity or porosity) nor the presence of unaccounted for chemically bound water can explain the departure of $S_{\text {eff }} / S_{\text {nom }}$ from unity.

Outstanding is the question of whether a temperature difference across the wetted blotting paper can account for the supersaturation discrepancy (i.e., $S_{\text {eff }} /$ $\left.S_{\text {nom }}<1\right)$. We address this by evaluating the temperature difference two different ways. In the first method 


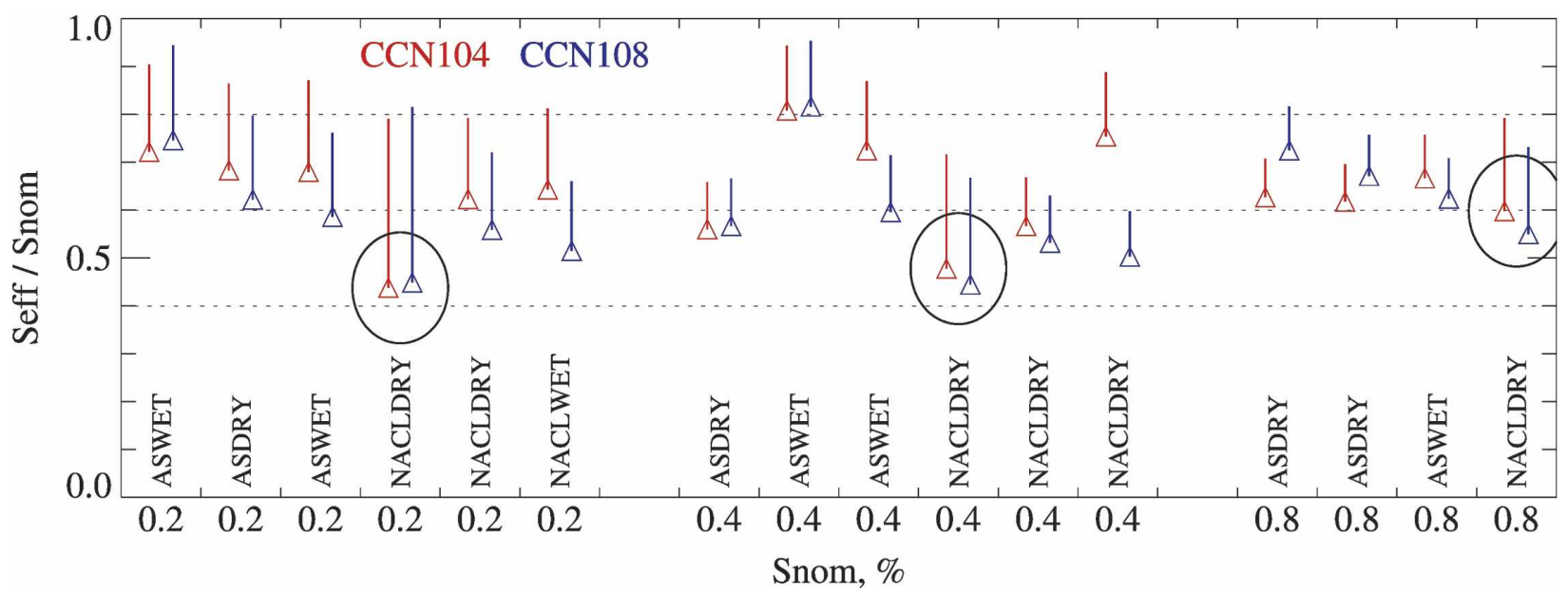

FIG. 11. Ratios of effective to nominal supersaturation (i.e., $S_{\text {eff }} / S_{\text {nom }}$ ) plotted vs nominal supersaturation. Results are stratified by $\mathrm{CCN}$ instrument, salt type, and particle selection technique. DYCOMS-II era results are circled. Upper-limit values indicated by the ends of the vertical lines are explained in the text.

the value of $S_{\text {eff }} / S_{\text {nom }}$ for CCN104 during HiCu (Table 4) and the chamber model were used to evaluate temperature differences that reconcile the supersaturation disparity. Values for a single piece of wetted blotting paper range between $0.2^{\circ}$ and $0.5^{\circ} \mathrm{C}$ for $S_{\text {nom }}=0.2 \%$ and $1.6 \%$, respectively. For the second method, we evaluate the temperature difference by setting the sum of the latent and sensible heat fluxes, again predicted by the chamber model, equal to the conductive heat flux across a single piece of wetted blotting paper. Values of the combined heat fluxes range between 19 and $50 \mathrm{~W}$ $\mathrm{m}^{-2}$ for $S_{\text {nom }}=0.2 \%$ and $1.6 \%$, respectively. A value of $0.42 \mathrm{~W}(\mathrm{~m} \mathrm{~K})^{-1}$ was adopted for the thermal conductivity of the wetted blotter paper, which was evaluated as a mass-weighted average of water and dry paper conductivities (Nenes et al. 2001) using a measured water-to-paper mass ratio for wetted paper (2:1). Values of the temperature difference corresponding to the second method were $26 \%$ of that anticipated by the first method. This demonstrates that the supersaturation discrepancy may be attributable, at least partially, to a temperature change from one side of the blotting paper to the other. These calculations also show that $S_{\text {nom }}$ converges to $S_{\text {eff }}$ when the conductivity of the wetted paper is taken to be that of dry paper $\left[0.1 \mathrm{~W}(\mathrm{~m} \mathrm{~K})^{-1}\right]$. However, this scenario seems unreasonable given that water is observed to be the dominant component of the wetted paper and that the wetted paper conductivity was calculated as a mass-weighted average.

We also evaluated the vapor pressure lowering, expressed as a water activity, that forces agreement between $S_{\text {eff }}$ and $S_{\text {nom. }}$. Derived water activity values range between 0.9994 and 0.9952 for $S_{\text {nom }}=0.2 \%$ and $1.6 \%$, respectively. The fact that a range of activities is re- quired to force agreement between $S_{\text {eff }}$ and $S_{\text {nom }}$ attenuates the likelihood that vapor pressure lowering is a viable explanation. Thus, neither of the two proposals-1) temperature change from one side of the paper to the other or 2) vapor pressure lowering - provides a persuasive explanation for the supersaturation discrepancy.

In Fig. 11 the DYCOMS-II era chamber calibrations are circled to point out that these are shifted to smaller $S_{\text {eff }} / S_{\text {nom }}$ values, particularly at $S_{\text {nom }}=0.2 \%$ and $0.4 \%$. The magnitude and direction of the shift, $\sim 35 \%$ below the $\mathrm{HiCu} S_{\text {eff }} / S_{\text {nom }}$ average at $S_{\text {nom }}=0.4 \%$, are consistent with the effect of a $0.5^{\circ} \mathrm{C}$ shift of $\Delta T$, that is, what we expected from a comparison of the DYCOMS-II and $\mathrm{HiCu}$ detector calibrations at $S_{\text {nom }}=0.4 \%$ ( $\mathrm{sec}$ tion $4 d)$.

The above discussions deal with biases that affect the

TABLE 4. Chamber calibration results.

\begin{tabular}{llccr}
\hline \hline Instrument & \multicolumn{1}{c}{ Era } & $\kappa_{50}{ }^{\mathrm{a}}$ & $\sigma_{\kappa}{ }^{\mathrm{b}}$ & $n$ \\
\hline CCN104 & DYCOMS-II & $0.50^{\mathrm{c}}$ & $\mathrm{NC}^{\mathrm{d}}$ & 3 \\
CCN108 & DYCOMS-II & $0.48^{\mathrm{c}}$ & $\mathrm{NC}^{\mathrm{d}}$ & 3 \\
CCN104 & $\mathrm{HiCu}$ & $0.67^{\mathrm{e}}$ & $0.06^{\mathrm{e}}$ & 13 \\
CCN108 & $\mathrm{HiCu}$ & $0.60^{\mathrm{e}}$ & $0.13^{\mathrm{e}}$ & 13 \\
\hline
\end{tabular}

${ }^{a}$ Values of $\kappa_{50}$, the best-fit value of $S_{\text {eff }} / S_{\text {nom }}$. These were fitted by forcing half of the data points to lie on or above the best-fit line. See text for details.

${ }^{\mathrm{b}} \sigma_{\kappa}$ is the random error associated with $\kappa_{50}$.

${ }^{\mathrm{c}}$ These are computed as averages of the three DYCOMS-II era $S_{\text {eff }} / S_{\text {nom }}$ ratios (circled in Fig. 11).

${ }^{\mathrm{d}} \mathrm{NC}=$ not calculated because the number of data points (3) is too small.

${ }^{\text {e }}$ Based on $S_{\text {eff }} / S_{\text {nom }}$ values and the percentile fitting technique described in section $4 \mathrm{~d}$. 
$S_{\text {eff }} / S_{\text {nom }}$ ratio and plausible reasons for a departure of that ratio from unity. In addition to bias, Fig. 11 also shows variability associated with the set of $S_{\text {eff }} / S_{\text {nom }}$ ratios; here we investigate that variability. When the $\mathrm{HiCu}$ era data are examined, and stratified by $S_{\text {nom, }}$, the $S_{\text {eff }} / S_{\text {nom }}$ standard deviation extends from relatively large values for measurements made at $S_{\text {nom }}=0.4 \%$, with standard deviations equal to 0.11 , to smaller variability for CCN104 at $S_{\text {nom }}=0.2 \%$ (standard deviation $=0.04$ ), compared to CCN108 at $S_{\text {nom }}=0.2 \%$ (standard deviation $=0.09)$, and for both instruments there is smaller variability at $S_{\text {nom }}=0.8 \%$ (standard deviation $=0.04)$. This variability can be traced to the detector calibration. Recall that we identified two processes that generate detector calibration uncertainty. One of these we quantified in terms of a systematic shift, from one instance of the detector calibration to another $\left(2 \delta_{\alpha}\right.$, section $\left.4 \mathrm{e}\right)$. We estimate the effect of this bias on $S_{\text {eff }} / S_{\text {nom }}$ by perturbing $\alpha_{50}$ over the range from $\alpha_{50}-2 \delta_{\alpha}$ to $\alpha_{50}+2 \delta_{\alpha}$. Our analysis involves an assessment of vertical shifts of the activation function, consistent with the supersaturation- and instrumentdependent values of $\delta_{\alpha}$ (Table 3 ), conversion of this to shifts in $D_{50}$, to shifts in $S_{\text {eff }}$ (via the Köhler model), and thus to shifts in $S_{\text {eff }} / S_{\text {nom }}$. In many instances, including results for CCN104 and CCN108 at $S_{\text {nom }}=0.2 \%$, this formulation can explain all of the $S_{\text {eff }} / S_{\text {nom }}$ variability. In other instances, and in particular for the results for $S_{\text {nom }}=0.4 \%$ (both instruments), only $30 \%$ of the observed variability can be explained. The residual variability is thought to be due to factors not accounted for in our error analysis or due to the limited number of individual $\alpha_{50}$ available for calculating $\delta_{\alpha}$ (Fig. 7 and Table 3).

We summarize the project- and instrument-dependent values of $S_{\text {eff }} / S_{\text {nom }}$ in Table 4 , where $S_{\text {eff }} / S_{\text {nom }}$ is symbolized as $\kappa_{50}$ and the $S_{\text {eff }} / S_{\text {nom }}$ standard deviation is symbolized as $\sigma_{\kappa}$. In the case of $\mathrm{HiCu}$, the value of $\kappa_{50}$ was fitted by plotting $S_{\text {eff }}$ versus $S_{\text {nom }}$ and by dictating a best-fit line of the form $S_{\text {eff }}=\kappa_{50} S_{\text {nom, }}$, subject to the constraint that half of the data points plot below it. The value of $\sigma_{\kappa}$ was constrained by dictating that $84 \%$ of the data lie below the line defined by $S_{\text {eff }}=\left(\kappa_{50}+\sigma_{\kappa}\right) S_{\text {nom }}$. This approach is the same as that used to define the detector calibration and its uncertainty. Because the number of $S_{\text {eff }} / S_{\text {nom }}$ measurement pairs is only three per instrument, the DYCOMS-II values of $\kappa_{50}$ are presented as $S_{\text {eff }} / S_{\text {nom }}$ averages. Plots of $S_{\text {eff }}$ versus $S_{\text {nom }}$ (not shown) showed no evidence of nonlinearity or a nonzero $y$-axis intercept.

Work conducted in the 1970s revealed discrepancies in the direction that we report in Table $4\left(S_{\text {eff }} / S_{\text {nom }}<1\right.$; Katz and Kocmond 1973) and also consistency among nominal and effective supersaturations (Gerber et al. 1977). Working with Wyoming CCN instruments, both Leaitch et al. (1999) and Bilde and Svenningsson (2004) reported effective and nominal supersaturations. The $S_{\text {eff }} / S_{\text {nom }}$ ratios reported in these two studies were 0.75 and 0.78 , respectively. The averaged ratios we present (Table 4) are somewhat smaller than those reported by either Leaitch et al. (1999) and Bilde and Svenningsson (2004). Previously, we reported $S_{\text {eff }} / S_{\text {nom }}$ ratios for CCN104 (Snider et al. 2003) based on the plate temperature calibration method used for DYCOMS-II and on an aerosol generation technique that differed slightly from that employed in this study (section 3c). For that dataset the averaged $S_{\text {eff }} / S_{\text {nom }}$ ratio was $0.6 \pm$ $0.1(n=7)$.

\section{Conclusions}

Our main result is that the maximum chamber supersaturation derived from experiments performed using test aerosols and a Köhler model departs from a supersaturation based on measured temperatures and a chamber model. Because there is uncertainty about parameters used to quantify the two mechanisms proposed for the supersaturation discrepancy (i.e., wetted paper thermal conductivity and water activity), we are not able to pinpoint its cause. Regardless of the reason for the discrepancy, it seems appropriate to use values of $S_{\text {eff }}$, rather than $S_{\text {nom }}$, in studies aimed at quantifying the CCN activity of either synthetic or ambient aerosols. Further, we develop techniques leading to five coefficients $\left(\alpha_{50}, \sigma_{\alpha}, \delta_{\alpha}, \kappa_{50}\right.$, and $\left.\sigma_{\kappa}\right)$. These describe the operation of the light scattering detection, thermal diffusion, Wyoming $\mathrm{CCN}$ instrument. When applied to raw data output by the instrument, in combination with a plate temperature calibration, these coefficients are sufficient for deriving $\mathrm{CCN}$ concentrations, $\mathrm{CCN}$ activity spectra, and the associated uncertainties.

Acknowledgments. This work was supported by NSF Grant ATM-0103951. Any opinions, findings, and conclusions and recommendations are those of the authors and do not necessarily reflect the views of the National Science Foundation.

\section{APPENDIX}

\section{Nomenclature}

A

$D$

$\mathrm{CCN}$ activation fraction

Particle diameter

Water vapor diffusivity

Water vapor supersaturation 


\begin{tabular}{|c|c|}
\hline $\begin{array}{l}\Delta T \\
\Delta_{\mathrm{DMA}}\end{array}$ & $\begin{array}{l}\text { Plate temperature difference } T_{t}-T_{b} \\
\text { Base width of the DMA transfer } \\
\text { function }\end{array}$ \\
\hline$\Delta a, \Delta b$ & $\begin{array}{l}\text { Difference of detector calibration fit- } \\
\text { ting coefficients }\end{array}$ \\
\hline & $\begin{array}{l}\text { Transformation from electrical mo- } \\
\text { bility to particle diameter }\end{array}$ \\
\hline & $\mathrm{CCN}$ detector sample volume \\
\hline & Electrical mobility \\
\hline$\alpha_{50}$ & Detector calibration coefficient \\
\hline$\kappa_{50}$ & Coefficient relating $S_{\text {eff }}$ to $S_{\text {nom }}$ \\
\hline$\sigma_{\alpha}$ and $\sigma_{\kappa}$ & $\begin{array}{l}\text { Standard deviation for distributions } \\
\text { of } \alpha_{50} \text { and } \kappa_{50} \text {, respectively }\end{array}$ \\
\hline$\sigma_{50}$ & $\begin{array}{l}\text { Standard deviation of the activation } \\
\text { function }\end{array}$ \\
\hline$\sigma_{\mathrm{DMA}}$ & $\begin{array}{l}\text { Standard deviation of singlet mode of } \\
\text { SMPS-derived size spectrum }\end{array}$ \\
\hline$\delta_{T}, 2 \delta_{50}$, and $2 \delta_{\alpha}$ & $\begin{array}{l}\text { Accuracy estimates for } \Delta T, D_{50} \text {, and } \\
\alpha_{50} \text {, respectively }\end{array}$ \\
\hline
\end{tabular}

\section{REFERENCES}

Bates, T. S., B. J. Huebert, J. L. Gras, F. B. Griffiths, and P. A. Durkee, 1998: International Global Atmospheric Chemistry (IGAC) Project's First Aerosol Characterization Experiment (ACE 1). Overview. J. Geophys. Res., 103, 16 297-16 318.

Bilde, M., and B. Svenningsson, 2004: CCN activation of slightly soluble organics: The importance of small amounts of inorganic salt and particle phase. Tellus, 56B, 128-134.

Brechtel, F. J., and S. M. Kreidenweis, 2000: Predicting particle critical supersaturation from hygroscopic growth measurements in the humified TDMA. Part II: Laboratory and ambient studies. J. Atmos. Sci., 57, 1872-1887.

Brenguier, J.-L., 2003: Introduction to Special Section: An experimental study of the aerosol indirect effect for validation of climate model parameterizations. J. Geophys. Res., 108, 8627, doi:10.1029/2003JD003849.

Burns, G. W., M. G. Scroger, G. F. Strouse, M. C. Coarkin, and W. F. Guthrie, 1993: Temperature-Electromotive Force Reference Functions and Tables for the Letter-Designated Thermocouple Types Based on ITS-90T. NIST Monogr., No. 175, NIST, 288 pp.

Chýlek, P., and J. G. D. Wong, 1998: Erroneous use of the modified Köhler equation in cloud an aerosol physics applications. J. Atmos. Sci., 55, 1473-1477.

Covert, D. S., J. L. Gras, A. Wiedensohler, and F. Stratmann, 1998: Comparisons of directly measured CCN and CCN modeled from the number-size distribution in the marine boundary layer during ACE 1 at Cape Grim, Tasmania. J. Geophys. Res., 103, 16 597-16 608.

Delene, D. J., and T. Deshler, 2000: Calibration of a photometric cloud condensation nucleus counter designed for deployment on a balloon package. J. Atmos. Oceanic Technol., 17, 459467.

Fitzgerald, J. W., 1972: On the computation of steady-state supersaturations in thermal diffusion chambers. J. Atmos. Sci., 29, 779-781.

Flagan, R. C., 1999: On differential mobility analyzer resolution. Aerosol Sci. Technol., 30, 556-570.

Gerber, H. E., W. A. Hoppel, and T. A. Wojciechowski, 1977:
Experimental verification of the theoretical relationship between size and critical supersaturation of salt nuclei. J. Atmos. Sci., 34, 1836-1841.

Gras, J. L., 1995: CN, CCN and particle size in the southern ocean air at Cape Grim. Atmos. Res., 35, 233-251.

Gysel, M., E. Weingartner, and U. Baltensperger, 2002: Hygroscopicity of aerosol particles at low temperatures. 2. Theoretical and experimental hygroscopic properties of laboratory generated aerosols. Environ. Sci. Technol., 36, 63-68.

Hinds, W. C., 1999: Aerosol Technology: Properties, Behavior, and Measurement of Airborne Particles. 2d ed. John Wiley \& Sons, $483 \mathrm{pp}$.

Katz, J. L., and P. Mirabel, 1975: Calculation of supersaturation profiles in thermal diffusion cloud chambers. J. Atmos. Sci., 32, 646-652.

Katz, U., and W. C. Kocmond, 1973: An investigation of the sizesupersaturation relationship of soluble condensation nuclei. J. Atmos. Sci., 30, 160-165.

Knutson, E. O., and K. T. Whitby, 1975: Aerosol classification by electric mobility: Apparatus, theory, and applications. J. Aerosol Sci., 6, 443-451.

Leaitch, W. R., J. W. Bottenheim, T. A. Biesenthal, S.-M. Li, P. S. K. Liu, K. Asalain, H. Dryfhouft-Clark, and F. Hopper, 1999: A case for gas-to-particle conversion in an eastern Canadian forest. J. Geophys. Res., 104, 8095-8111.

Lewis, E. R., and S. E. Schwartz, 2004: Sea Salt Aerosol Production: Mechanisms, Methods, Measurements and Models-A Critical Review. Geophys. Monogr., No. 152, Amer. Geophys. Union, $412 \mathrm{pp}$.

Nenes, A., P. Y. Chuang, R. C. Flagan, and J. H. Seinfeld, 2001: A theoretical analysis of cloud condensation nucleus $(\mathrm{CCN})$ instruments. J. Geophys. Res., 106, 3449-3474.

Oliveira, J. C. P., and G. Vali, 1995: Calibration of a photoelectric cloud condensation nucleus counter. Atmos. Res., 38, $237-$ 248

Rissler, J., E. Swietlicki, J. Zhou, G. Roberts, M. O. Andreae, L. V. Gatti, and P. Artaxo, 2004: Physical properties of the sub-micrometer aerosol over the Amazon rain forest during the wet-to-dry season transition-Comparison of modeled and measured CCN concentrations. Atmos. Chem. Phys., 4, 2119-2143

Roberts, G. C., and A. Nenes, 2005: A continuous-flow streamwise thermal-gradient $\mathrm{CCN}$ chamber for atmospheric measurements. Aerosol Sci. Technol., 39, 206-221.

Rogers, R. R., and M. K. Yau, 1989: A Short Course in Cloud Physics. Elsevier Scientific, 290 pp.

Saxena, V. K., J. N. Burford, and J. L. Kassner Jr., 1970: Operation of a thermal diffusion chamber for measurements of cloud condensation nuclei. J. Atmos. Sci., 27, 73-80.

Seinfeld, J. H., and S. N. Pandis, 1998: Atmospheric Chemistry and Physics. John Wiley and Sons, 1326 pp.

Snider, J. R., S. Guibert, J.-L. Brenguier, and J.-P. Putaud, 2003: Aerosol activation in marine stratocumulus clouds: 2. Köhler and parcel theory closure studies. J. Geophys. Res., 108, 8629, doi:10.1029/2002JD002692.

Stevens, B., and Coauthors, 2003: Dynamics and chemistry of marine stratocumulus-DYCOMS-II. Bull. Amer. Meteor. Soc., 84, 579-593.

Tang, I. N., and H. R. Munkelwitz, 1994: Water activities, densities, and refractive indices of aqueous sulfates and sodium nitrate droplets of atmospheric importance. J. Geophys. Res., 99, 18 801-18 808.

Vaisala, 2001: HMP230 series transmitters user's guide. 155 pp. 